Ramarson Herintsitohaina RAZAKAMANARIVO ${ }^{1,2}$ Marie-Antoinette RAZAFINDRAKOTO ${ }^{2}$ Alain ALBRECHT ${ }^{1}$

${ }^{1}$ Laboratoire des radioisotopes (Lri)-Ird Umr 210 Eco\&Sols

BP 3383

Route d'Andraisoro 101

Antananarivo

Madagascar

2 École supérieure des sciences agronomiques Département Eaux et forêts

Université d'Antananarivo 101

BP 175

Antananarivo

Madagascar

\title{
Fonction puits de carbone des taillis d'eucalyptus à Madagascar
}

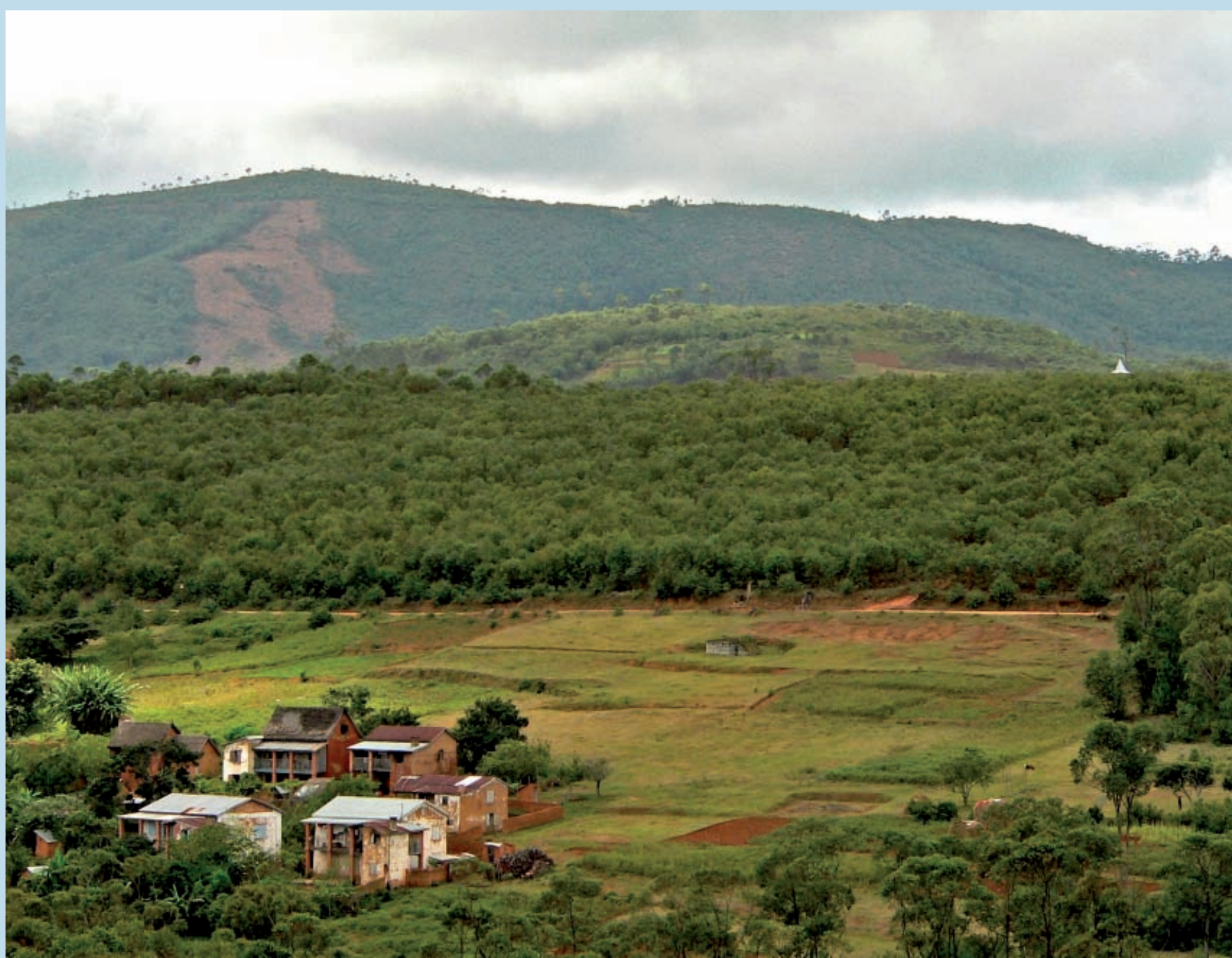

Photo 1.

Importance des plantations d'Eucalyptus robusta dominant le paysage des Hautes Terres malgaches. Ici (Sambaina-

Manjakandriana), les taillis d'eucalyptus se trouvent sur tous les types de versants, en bordure de route et aussi sur les collines. Photo C. Grinand. 


\section{RESUMÉ}

\section{FONCTION PUITS DE CARBONE DES TAILLIS D'EUCALYPTUS À MADAGASCAR}

Les écosystèmes forestiers jouent un grand rôle face au réchauffement climatique grâce aux réservoirs importants de carbone organique (C) de leurs compartiments (aérien, racinaire, litière et sol). En ce qui concerne l'utilisation des terres, les changements d'affectation des terres et la foresterie, des mesures précises des stocks de $C$ dans ces compartiments sont recommandées. Dans ce sens, la capacité de fixation de $\mathrm{C}$ des taillis d'eucalyptus (20 à 111 ans) des Hautes Terres centrales malgaches dans ces compartiments a été étudiée, en comparaison avec d'autres modes d'usage des terres (pseudo-steppe, rotation culture/jachère). Des mesures destructives de biomasses et des prélèvements de sols ont été effectués, suivis par le développement d'équations allométriques et des analyses en laboratoire (détermination de la teneur en carbone, étude isotopique). Tous âges confondus, la pratique de taillis d'eucalyptus présente le plus grand stock de $\mathrm{C}(150,8 \pm 25,3 \mathrm{Mg} \mathrm{C} / \mathrm{ha}$ face à $112 \pm 15$ et $72,3 \pm 9,9 \mathrm{Mg}$ C/ha pour la pseudo-steppe et la rotation culture/jachère respectivement). Pour tous les modes d'usage, c'est le compartiment sol qui constitue le principal réservoir de $C$ avec $56 \%$ pour l'eucalyptus et plus de $96 \%$ pour les autres modes. Mais ce sont essentiellement la souche et les racines lignifiées qui rendent ces plantations d'eucalyptus plus efficaces que les autres modes d'usage (44 Mg C/ha pour l'eucalyptus face à $2 \mathrm{Mg} \mathrm{C} /$ ha) en termes de stockage de C. D'après l'étude isotopique, le $\mathrm{C}$ dérivé de l'eucalyptus dans le sol ne domine toutefois qu'en surface, probablement en raison de la gestion en taillis. Cependant, d'autres facteurs, à part la durée de plantation, comme l'altitude et la morphopédologie sont à considérer pour comprendre la dynamique des stocks de $\mathrm{C}$ mesurés.

Mots-clés : carbone organique du sol, isotope ${ }^{13} \mathrm{C}$, biomasse racinaire, changement d'usage des terres, chronoséquence, séquestration de carbone, Madagascar.

\section{ABSTRACT}

\section{EUCALYPTUS COPPICE PLANTATIONS AS CARBON SINKS IN MADAGASCAR}

Forest ecosystems have an important role in helping to fight climate change because of their large potential for storing organic carbon (C) in their different compartments (aerial parts, roots, litter and soil). Concerning land use change and forestry, the recommendation is to make exact measurements of carbon stocks in these compartments. A study was made for this purpose, to determine the carbon-fixing capacities of eucalyptus coppice plantations (20 to 111 years old) in the Central Uplands of Madagascar, in comparison with other types of land use (pseudo-steppe, crop/fallow rotation). Following destructive biomass measurements and soil sampling, allometric equations were developed and laboratory analyses performed (determination of carbon content, isotopic analyses). Taking all tree ages together, eucalyptus coppices contain the largest stocks of carbon $(150.8 \pm 25.3 \mathrm{MgC} / \mathrm{ha}$ as against $112 \pm 15$ and $72.3 \pm 9.9 \mathrm{MgC} /$ ha for pseudo-steppe and crop/fallow rotation respectively). Among all use types, the soil compartment is the largest carbon reservoir, with $56 \%$ in eucalyptus stands and over $96 \%$ in the other cases. These eucalyptus plantations store carbon more efficiently than other land uses (44 MgC/ha for eucalyptus as against $2 \mathrm{MgC} / \mathrm{ha}$ ) mainly thanks to the stump and lignified roots of the trees. However, according to the isotopic analysis, soil carbon derived from eucalyptus is only dominant on the surface, probably because of the coppice management method. Other factors besides plantation age, such as altitude and soil morphology, also need to be considered to understand the dynamics of the carbon stocks measured.

Keywords: organic carbon in soils, isotope ${ }^{13} \mathrm{C}$, root system biomass, land use change, chronosequence, carbon sequestration, Madagascar.

\section{RESUMEN}

\section{FUNCIÓN SECUESTRO DE CARBONO DE TALLARES DE EUCALIPTO EN MADAGASCAR}

Los ecosistemas forestales desempeñan un gran papel frente al cambio climático gracias a las importantes reservas de carbono orgánico (C) de sus compartimentos (aéreo, radicular, hojarasca y suelo). En el ámbito del uso de la tierra, cambios de uso de la tierra y silvicultura, se recomienda efectuar mediciones precisas de las reservas de $\mathrm{C}$ en estos compartimentos. En este sentido, se estudió la capacidad de fijación de $C$ en los compartimentos de tallares en renuevo de cepa de eucalipto (20 a 111 años) de las Tierras Altas Centrales de Madagascar, comparándola con la de otros modos de uso de la tierra (pseudoestepa, rotación de cultivos/barbecho). Se efectuaron medidas destructivas de biomasa y muestreo de suelos para, seguidamente, desarrollar ecuaciones alométricas y realizar análisis en laboratorios (determinación del contenido de carbono, estudio isotópico). Sin distinción de edad, el tratamiento de renuevos de cepa de eucalipto presenta la mayor acumulación de C $(150.8 \pm 25.3 \mathrm{MgC} /$ ha frente a $112 \pm 15$ y $72.3 \pm 9.9 \mathrm{MgC} /$ ha para la pseudoestepa y la rotación de cultivos/barbecho, respectivamente). En todas las formas de uso, el suelo es el compartimento que constituye el principal depósito de C, con el $56 \%$ para el eucalipto y más del $96 \%$ para los demás modos. Pero, sobre todo, son las cepas y las raíces lignificadas las que hacen que estas plantaciones de eucalipto sean más eficaces en términos de secuestro de $C$ que los otros modos de uso (44 MgC/ha para al eucalipto frente a $2 \mathrm{MgC} / \mathrm{ha}$ ). Sin embargo, según el estudio isotópico, el $\mathrm{C}$ en el suelo procedente del eucalipto, sólo domina en la superficie, probablemente debido al manejo en tallar bajo. No obstante, además de la duración de la plantación, se deben considerar otros factores como la altitud y la morfoedafología para comprender la dinámica del estoc de $\mathrm{C}$ que se mide.

Palabras clave: carbono orgánico del suelo, isótopo ${ }^{13} \mathrm{C}$, biomasa radicular, cambio de uso de las tierras, cronosecuencia, secuestro de carbono, Madagascar. 


\section{Introduction}

\section{Contexte scientifique}

Avec les préoccupations actuelles concernant le réchauffement global lié à l'augmentation des teneurs en gaz à effet de serre ou Ges (gaz carbonique $\mathrm{CO}_{2}$, méthane $\mathrm{CH}_{4}$, oxyde nitreux $\mathrm{N}_{2} \mathrm{O}$ ) de l'atmosphère, les écosystèmes terrestres, en particulier forestiers, jouent un rôle important dans l'atténuation de ce phénomène climatique. En effet, avec des réservoirs estimés à 652 gigatonnes de carbone (Gt C) pour la végétation et 1500 à 2000 Gt C pour le sol (jusqu'à un mètre de profondeur) (FAO, 2001 ; IPCC, 2007), ces écosystèmes ont une grande influence sur le cycle du carbone (C) (Locatelli, Lescuyer, 1999 ; Robert, SAugier, 2003). Tout changement de mode d'usage des terres peut avoir par conséquent des effets considérables sur leur capacité de séquestrer du carbone (Post, KNOWN, 2000 ; GUO, GIFFORD, 2002). II s'avère ainsi indispensable de connaître les potentialités de chaque catégorie d'affectation des terres (culture, savane, pâture, plantations forestières, forêts naturelles, etc.) en termes de séquestration de $C$ et de pouvoir les gérer. Pour cela, plusieurs compartiments qui contribuent à fixer le $\mathrm{C}$ atmosphérique sont à considérer (IPCC, 2006), telles les biomasses aérienne et racinaire, la matière organique morte de la litière et la matière organique du sol. En général, le stock de $\mathrm{C}$ dans ces différents compartiments des plantations forestières augmente avec leur âge (PAUL et al., 2002 ; LAL, 2005). Toutefois, divers facteurs peuvent affecter cette capacité de fixation de C (LoCATELLI, LeSCUYER, 1999 ; Guo, GIFFORD, 2002), tels l'espèce, les soins sylvicoles, la durée de rotation, l'antécédent cultural.

Concernant les Ges non $\mathrm{CO}_{2}$, ce sont surtout les sols des forêts tempérées qui constituent un puits important de $\mathrm{CH}_{4}$ et une source d'un peu de $\mathrm{N}_{2} \mathrm{O}$ lors du recyclage des nutriments (DALAL et al., 2008 ; LIVESLEY et al., 2009). Toutefois, des études sur des peuplements d'eucalyptus ont conclu que c'est le $\mathrm{CO}_{2}$ qui constitue le principal Ges concerné dans ces écosystèmes, les flux des deux autres gaz étant faibles. FEST et al. (2009) ont montré qu'une forêt d'Eucalyptus delegatensis en Australie constitue un puits net de Ges, avec une contribution de seulement $0,3 \%$ de la fixation de $\mathrm{CH}_{4}$ et de $0,3 \%$ de l'émission de $\mathrm{N}_{2} \mathrm{O}$.

À Madagascar, l'eucalyptus est une espèce massivement adoptée par les populations malgaches en raison de son ancienne diffusion, de sa capacité à constituer une alternative à la dégradation des forêts naturelles, mais aussi pour l'appropriation foncière (CARRIÈRE, RANDRIAMBANONA, 2007). Couvrant plus de 150000 hectares (ha) en 2005 (MeYers et al., 2006), les plantations d'eucalyptus sont localisées majoritairement sur les Hautes Terres centrales ou Htc (photo 2) et tiennent une place importante chez les communautés malgaches : elles fournissent du bois de chauffe, du bois de construction et du charbon de bois, notamment pour les milieux urbains, et les revenus réguliers qu'elles génèrent contribuent à l'amélioration des conditions de vie des communautés rurales. Toutefois, leur potentiel en termes de séquestration de $\mathrm{C}$ reste encore méconnu, alors que ces pratiques forestières pourraient être valorisées en termes de crédits carbone qui généreront des revenus via le marché du carbone à travers les projets du Mécanisme de développement propre (Mdp), dans le cadre du protocole de Kyoto (1997) de la Convention-cadre des Nations unies sur le changement climatique. En effet, les taillis d'eucalyptus des Htc malgaches présentent des caractéristiques particulières, contrairement aux plantations d'eucalyptus étudiées généralement dans la littérature. Si, dans ces derniers cas, les plantations sont souvent industrialisées et gérées en futaie, sur les Htc malgaches, elles sont caractérisées par un mode de gestion contraignant (courte rotation, pas de fertilisation ni de soins sylvicoles), des souches très âgées et un contexte paysan.

L'objectif de cette étude est de quantifier la contribution réelle des plantations d'eucalyptus à la constitution du stock de $C$ dans les différents compartiments (aérien, racinaire, litière, matière organique du sol) au regard du mode de gestion et de comparer la capacité des vieilles souches à fixer du C avec deux autres modes d'usage des terres: la pseudosteppe (système originel) et la rotation culture/jachère.

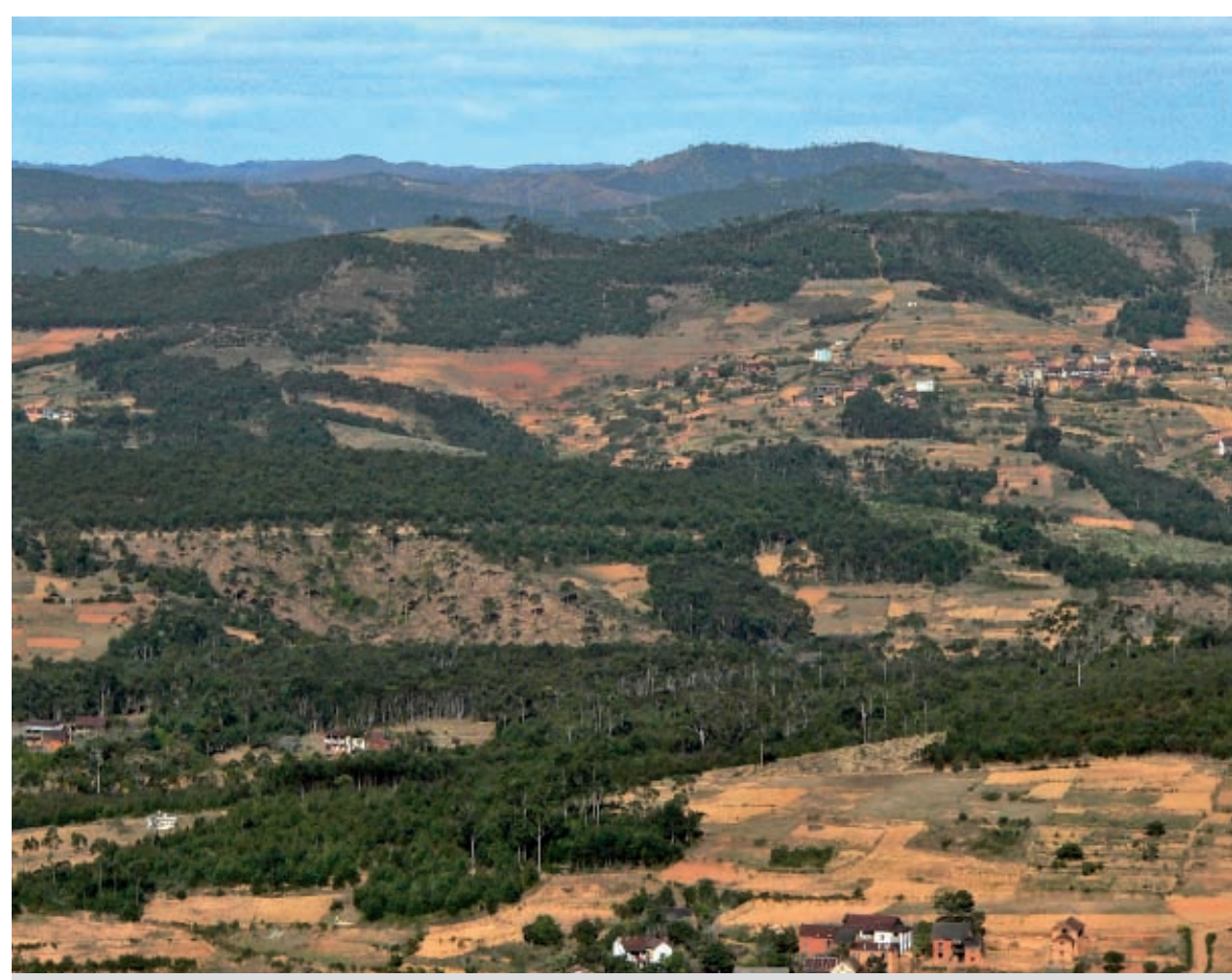

Photo 2.

Divers types de reliefs et de versants sont recouverts de plantations d'eucalyptus dans la commune rurale de Sambaina-Manjakandriana; une saturation de l'espace est observée. Photo R. H. Razakamanarivo. 
BOIS ET FORÊTS DES TROPIQUES, $2010, N^{\circ} 305$ (3)

CARBON STOCK AND PLANTATIONS
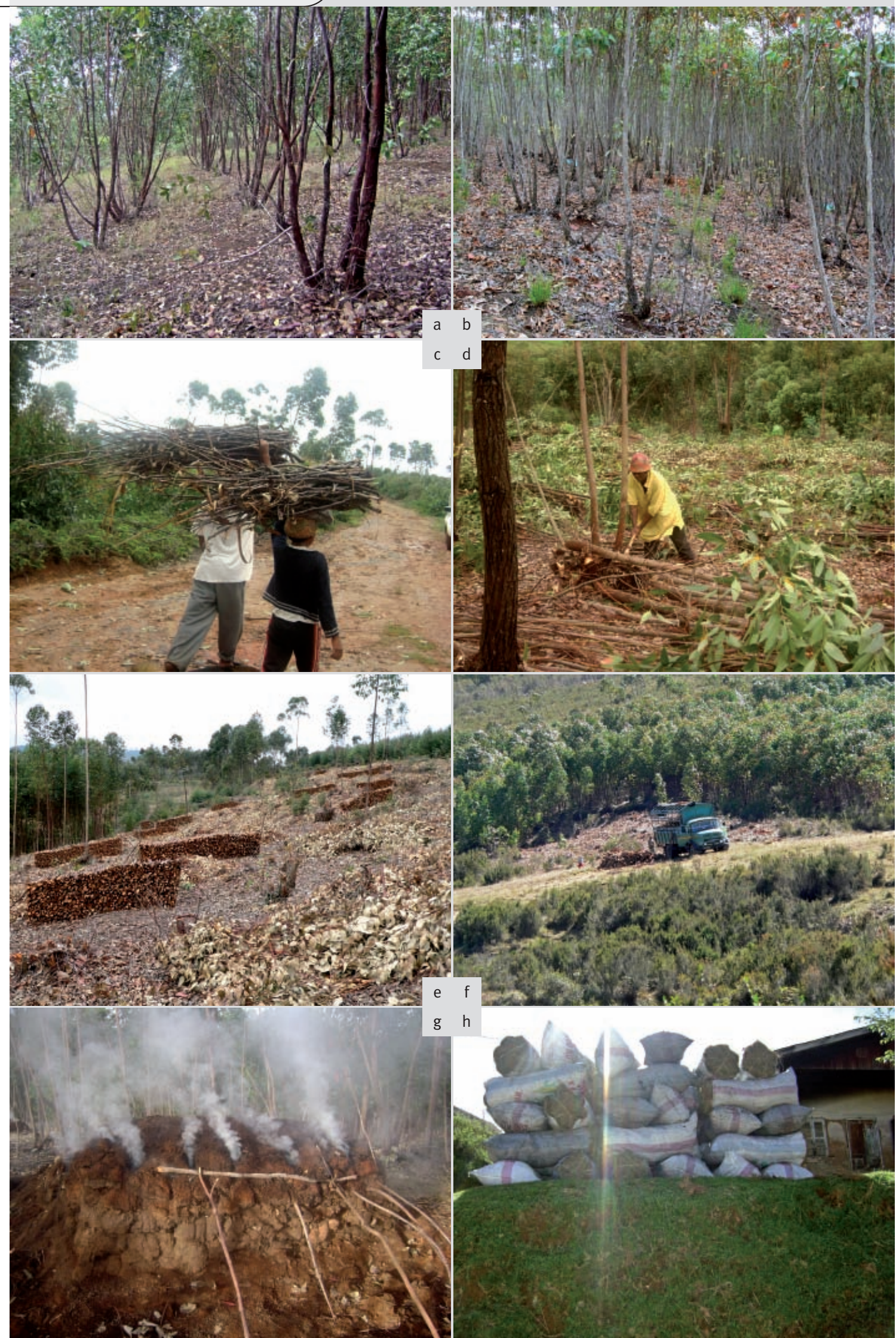

$\mathrm{g} h$
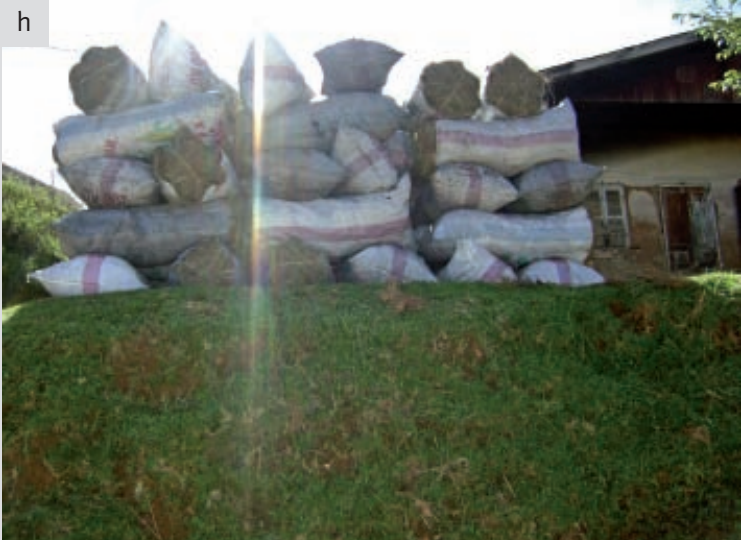

Photos 3.

Aperçu des taillis d'eucalyptus des Hautes Terres centrales malgaches et illustrations de leur utilisation : (a) et (b) parcelles de taillis en fin de rotation qui seront prochainement recépées; (c) enfants villageois transportant des branches d'eucalyptus qu'ils ont ramassées pour leur consommation locale de bois de chauffe et d'énergie ; (d) recépage d'une parcelle en fin de rotation assuré par un bûcheron du village ; (e) stocks de bois de chauffe et d'énergie récemment coupés sur la parcelle ; (f) chargement en bois de chauffe et d'énergie d'un camion assurant l'approvisionnement de la capitale ;

(g) charbonnière traditionnelle en cuisson au sein de la parcelle; (h) sacs de charbon prêts pour embarquement vers la ville. Photos R. H. Razakamanarivo. 


\section{Matériels et méthodes}

\section{Les plantations d'eucalyptus à Madagascar}

Les premières introductions de l'eucalyptus à Madagascar datent du début des années 1900, sur des terrains de savane et/ou pseudo-steppe. Avec une soixantaine d'espèces introduites à l'époque (GUÉNEAU, 1969 ; CARRIÈrE, RANDRIAMBANONA, 2007), les superficies plantées n'ont cessé de s'accroître, constituant ainsi une chronoséquence. Parmi les 180 espèces d'eucalyptus introduites, douze ont été prouvées comme prometteuses pour la production de bois d'œuvre, d'industrie et d'énergie : Eucalyptus robusta, E. camaldulensis, E. grandis, E. citriodora, E. cloeziana, E. corymbosa, E. eugenioides, E. macrorhyncha, E. maculata, E. microcorys, E. tereticornys et E. torelliana (RANDRIANJAFY, 1999). Mais $E$. robusta a marqué une extension particulière grâce à sa faculté d'adaptation et son aptitude à pousser avec vigueur sur des sols compacts, tout en résistant au feu. L'aptitude à rejeter des souches d'E. robusta permet des coupes régulières (régime sylvicole de taillis) pour la production de charbon et de bois de chauffe (photos 3). La durée de rotation est courte, allant de 3 à 5 ans, selon les besoins financiers des propriétaires. En général, les pieds ne sont pas renouvelés depuis leur mise en place, les peuplements restent livrés à eux-mêmes (pas d'entretien sylvicole) et la litière de feuilles décomposées d'eucalyptus (localement appelée fongo) (photos 4) se récolte et parfois se vend comme engrais de complément (BERTRAND, 1999). Sous forme de fumure organique (litière mélangée avec du fumier, des terreaux et des déchets ménagers et agricoles), le fongo est épandu dans les rizières ou sur les versants cultivés.

\section{Sites d'étude}

Deux communes rurales appartenant au district de Manjakandriana ont été choisies : l'une parce qu'elle témoigne de l'importance ancienne des plantations d'eucalyptus dans l'ensemble de son territoire (cas de la commune de Sambaina), l'autre parce qu'elle présente un front de boisement (cas de la commune de Sadabe).

Le climat du district de Manjakandriana est de type tropical d'altitude avec une température moyenne annuelle de $17^{\circ} \mathrm{C}$ et une pluviosité moyenne annuelle de $1618 \mathrm{~mm}$. La roche mère est constituée de granite porphyroïde. Les sols sont généralement des ferralsols (FAO, 2006), localement appelés sols ferrallitiques jaune sur rouge (BouRGEAT, ZEBROWSKI, 1973) (photo 5).

La commune rurale de Sambaina, qui se trouve à $53 \mathrm{~km}$ au nord d'Antananarivo, couvre une superficie de 2900 ha entre les latitudes $18^{\circ} 51^{\prime}$ et $18^{\circ} 56^{\prime}$ Sud et les longitudes $47^{\circ} 45^{\prime}$ et $47^{\circ} 48^{\prime}$ 'Est. L'altitude varie entre 1350 et $1750 \mathrm{~m}$. Mis à part les peuplements d'eucalyptus occupant plus de la moitié de la surface de la commune, deux autres modes d'usage sont présents : la pseudo-steppe constituée par une savane herbeuse (peuplée par Aristida sp. ou Imperata sp., Loudetia sp.) comportant des arbustes parsemés (Aphloia theaeformis, Psiadia altissima, Helichrysum sp., Phillipia sp.) et la rotation culture/jachère. La commune de Sadabe se trouve à $45 \mathrm{~km}$ au nord-est d'Antananarivo, occupant une superficie de 23150 ha entre les latitudes $18^{\circ} 30^{\prime}$ et $18^{\circ} 40^{\prime}$ Sud et les longitudes $47^{\circ} 41^{\prime}$ et $47^{\circ} 45^{\prime}$ 'Est. L'altitude y est de 1200 à 1500 m. Ce sont les savanes herbacées (composées
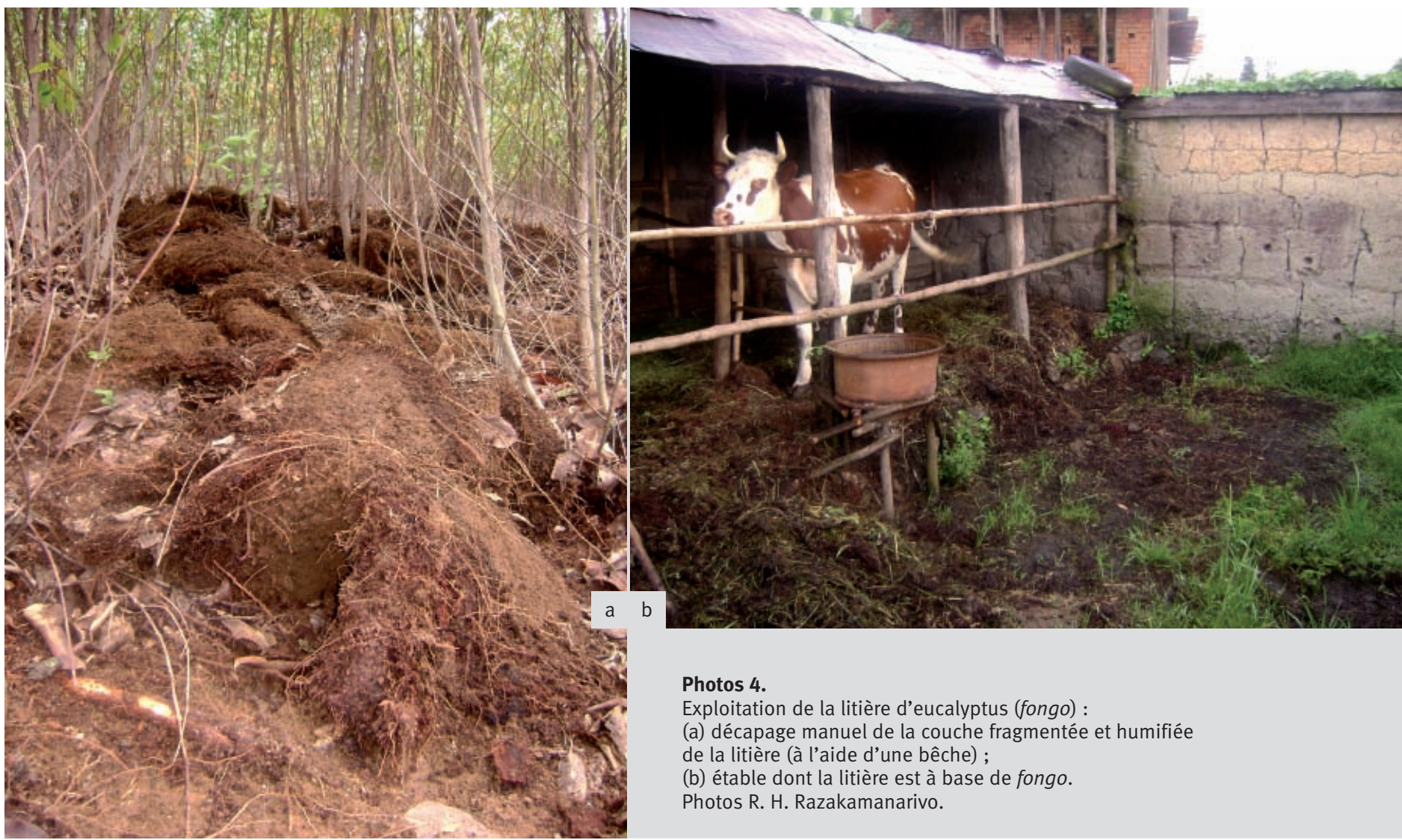

Photos 4.

Exploitation de la litière d'eucalyptus (fongo) :

(a) décapage manuel de la couche fragmentée et humifiée

de la litière (à l'aide d'une bêche) ;

(b) étable dont la litière est à base de fongo.

Photos R. H. Razakamanarivo. 


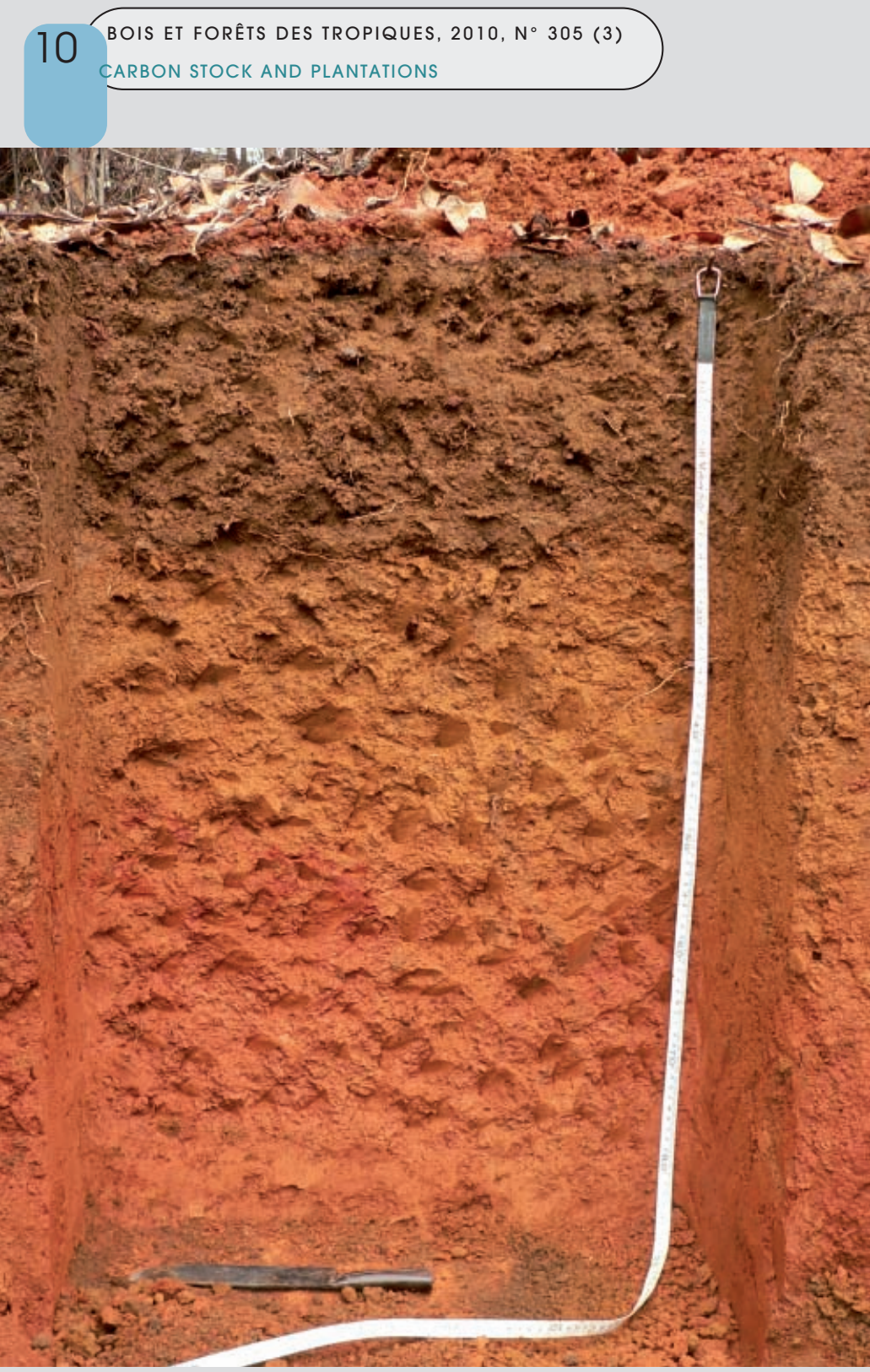

Photo 5.

Un profil du type de sol (jaune sur rouge) rencontré dans les zones d'étude sur une profondeur d'un mètre. Photo R. H. Razakamanarivo.

de Aristida sp. et Imperata sp.) qui dominent le paysage (plus de $53 \%$ ), les plantations d'eucalyptus mélangées à la savane arborée couvrant environ $35 \%$ de sa superficie (carte 1).

Trois principaux modes d'usage des terres sur les Htc de Madagascar ont été considérés : la pseudo-steppe (système originel précédant tout autre mode d'usage), la rotation culture/jachère (photos 6) et la chronoséquence d'eucalyptus. L'identification d'un certain nombre de parcelles pour chaque catégorie d'affectation des terres (tableau I) a été effectuée, en s'appuyant sur la photo-interprétation des photographies aériennes disponibles (en date de 1949, 1965 et 1995), par la suite combinée aux traitements d'images satellitaires (Quickbird et Spot 2006) et à des enquêtes sur le terrain, le tout intégré dans un système d’information géographique.

\section{Quantification des stocks de carbone}

\section{Biomasses aérienne et racinaire}

Pour les eucalyptus, les équations allométriques couramment utilisées dans la quantification de la biomasse en foresterie (LoCATELLI, LesCuYer, 1999 ; Peltier et al., 2007) ont été adoptées. Des équations ont été établies (tableau II) à l'aide de mesures destructives réalisées sur 27 souches. Ces équations lient le poids de la biomasse (matière sèche obtenue après étuvage à $70^{\circ} \mathrm{C}$ ) de chaque compartiment de l'arbre (aérien ou racinaire) avec une variable dendrométrique facilement mesurable sur le terrain, entre autres la circonférence. Pour la partie racinaire, contrairement à d'autres études qui estiment indirectement la biomasse en utilisant un coefficient appliqué à la biomasse aérienne (ratio aérien/racinaire ou A/R) (LOCATELLI, LESCUYER, 1999), les mesures ont été réalisées en adoptant le protocole du polygone de Voronoï. Ce protocole consiste à excaver toutes les racines se trouvant dans la surface de ce polygone (espace défini par la moitié des distances de l'arbre échantillonné à ses voisins ; SANTANTONIO et al., 1977), et ce jusqu'à 1 mètre de profondeur (photo 7). Les détails sur cette estimation de biomasse racinaire ont fait l'objet d'un article en amont de la présente étude.

La densité de biomasse aérienne ou racinaire à l'hectare a été ensuite calculée en appliquant ces équations allométriques établies aux mesures obtenues par inventaire.

Pour la litière, deux couches ont été considérées : la litière de l'année $\left(\mathrm{O}_{\mathrm{l}}\right)$ constituée par les débris de végétaux facilement reconnaissables et la couche fragmentée et humifiée $\left(\mathrm{O}_{f}+\mathrm{O}_{h}\right)$ mélangée au mat racinaire (qui constitue le fongo). Toute la litière contenue dans un cadre métallique (0,44 m x 0,44 m avec 3 ou 4 répétitions par parcelle) a été récoltée, pesée, et sa quantité de matière sèche estimée par échantillonnage et étuvage.

Pour les autres modes d'usage des terres, toute la matière fraîche au-dessus du sol se trouvant dans une surface définie $(0,5 \mathrm{~m}$ x 0,5 $\mathrm{m}$ avec 4 répétitions) a été prélevée, pesée et échantillonnée afin de déterminer la teneur en matière sèche. La partie racinaire a été extraite complètement jusqu'à $30 \mathrm{~cm}$ de profondeur.

Comme généralement considéré dans les études de quantification du $\mathrm{C}$ de la biomasse forestière, le coefficient par défaut de 0,5 a été utilisé pour calculer la quantité de C renfermée dans les biomasses mesurées (LoCATELLI, LESCUYER, 1999).

\section{Matière organique du sol \\ Quantification du stock de carbone du sol $\left(\mathrm{C}_{\mathrm{so}}\right)$}

Les échantillons de sol ont été prélevés à l'aide d'un cylindre métallique (volume de $528 \mathrm{~cm}^{3}$ ) sur quatre profondeurs (tous les $10 \mathrm{~cm}$ jusqu'à $40 \mathrm{~cm}$ de profondeur) pour quatre points de prélèvement par parcelle. Chaque échantillon a été divisé en deux : une aliquote destinée à la mesure de la teneur en humidité du sol et un reste, réservé à l'analyse chimique. La densité apparente (Da : masse de sol sec pour un volume donné) a donc été déterminée à partir de la teneur en humidité de l'aliquote après un passage à l'étuve à $105^{\circ} \mathrm{C}$. Le reste de sol brut prélevé a été séché à l'air libre et tamisé avec une maille de $2 \mathrm{~mm}$ (pour séparer les fractions gros- 


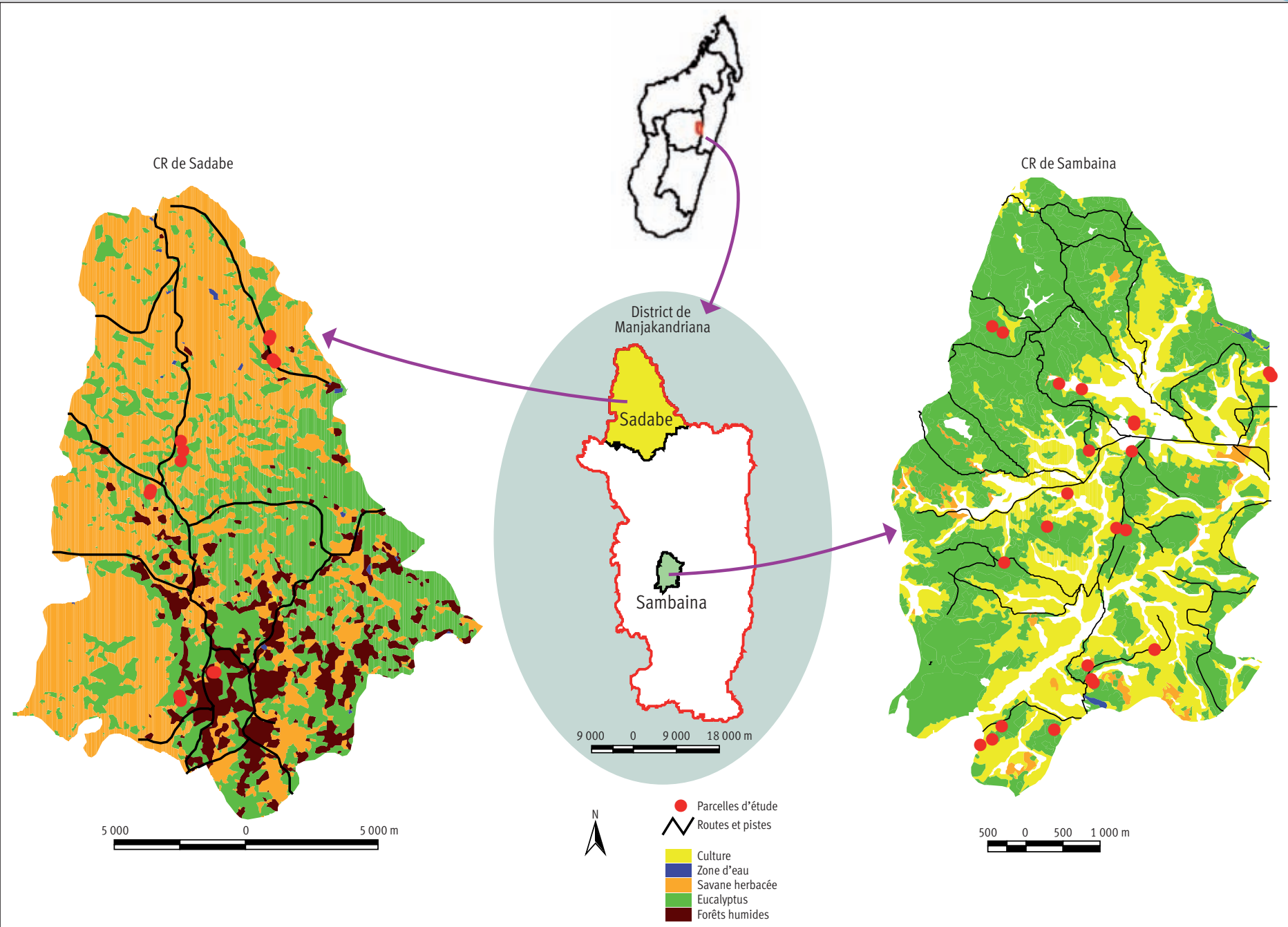

Carte 1.

Localisation des communes rurales étudiées et leur occupation des terres par rapport à la carte de Madagascar.
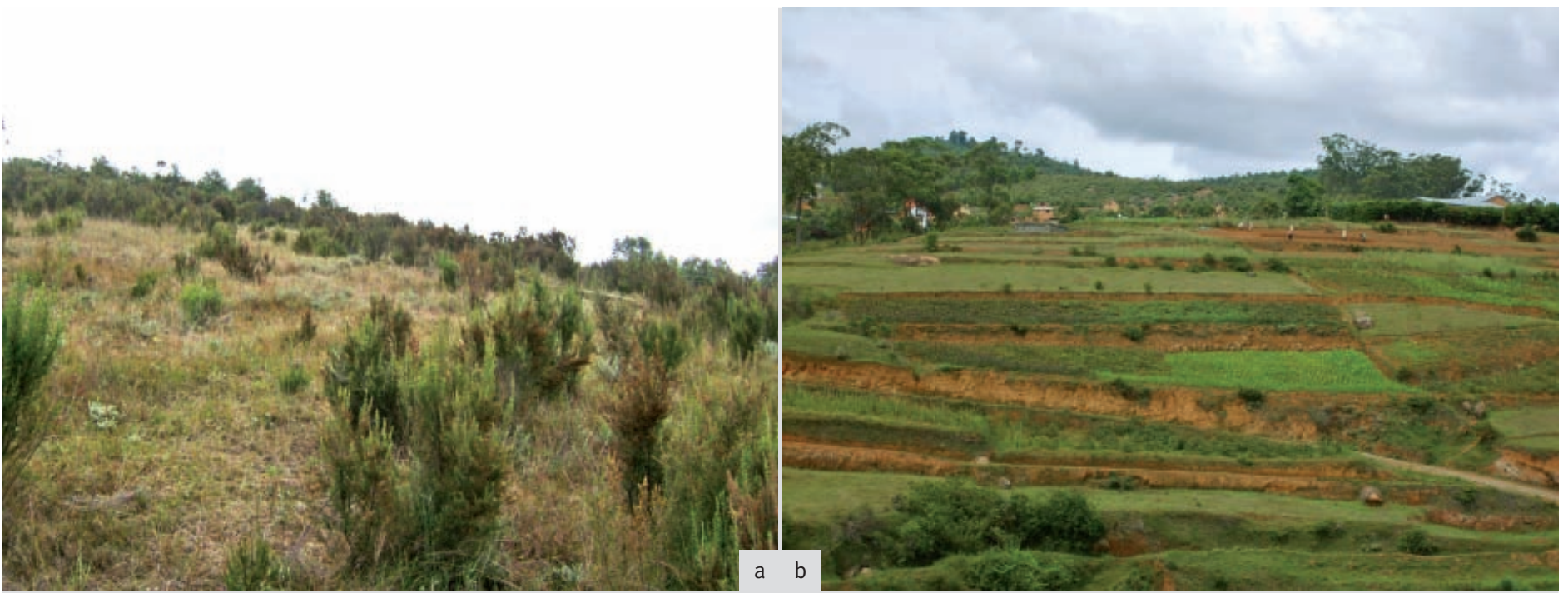

Photos 6.

Les autres modes d'usage des terres (autres que les taillis d'eucalyptus) étudiés : (a) pseudo-steppe avec les pieds de Philippia sp. ;

(b) diverses parcelles de rotation culture (au premier plan) et jachère (en second plan).

Photos R. H. Razakamanarivo. 
Tableau I.

Description des parcelles étudiées sur les Hautes Terres centrales de Madagascar.

Mode d'usage
$\begin{aligned} & \text { Plantation d'eucalyptus } \\ & \mathrm{N}=45\end{aligned}$
Culture/jachère
$\mathrm{N}=5$

Savane/pseudo-steppe $\mathrm{N}=7$

\section{Espèces}

Eucalyptus robusta

Manioc (Manihot esculenta), patate douce (Ipomoea batatas)

Imperata cylindrica

Aristida adescensionis

Philipia sp.

Hyparrhenia sp.

\section{Observations}

Propriétés privées (héritage familial) à surface variable : de quelques ares à une dizaine d'hectares, respectivement pour les familles moins aisées et plus aisées.

Durée de plantation : 19 à 111 ans avec 1500 à 6300 souches/ ha ; circonférence moyenne des souches (Cir), 141,2 cm.

Âge des rejets : 3 à 5 ans avec 7300 à 31100 rejets/ha et en moyenne 6 rejets par souche ; $8,2 \mathrm{~cm}$ de circonférence à hauteur de poitrine (Chp) et 4,2 $\mathrm{m}$ de hauteur totale $(\mathrm{H})$ pour les rejets.

En général, 3 ans de culture et 1 à 2 ans de jachère selon la disponibilité en terrain du propriétaire.

En général, la densité maximale des arbustes est de $10 \%$.

$\mathrm{N}$ : effectif de l'échantillon.

\section{Tableau II. Caractéristiques des équations de régression utilisées lors de la quantification de la biomasse des plantations d'eucalyptus des Hautes Terres centrales de Madagascar ( $\mathrm{N}=27$ arbres).}

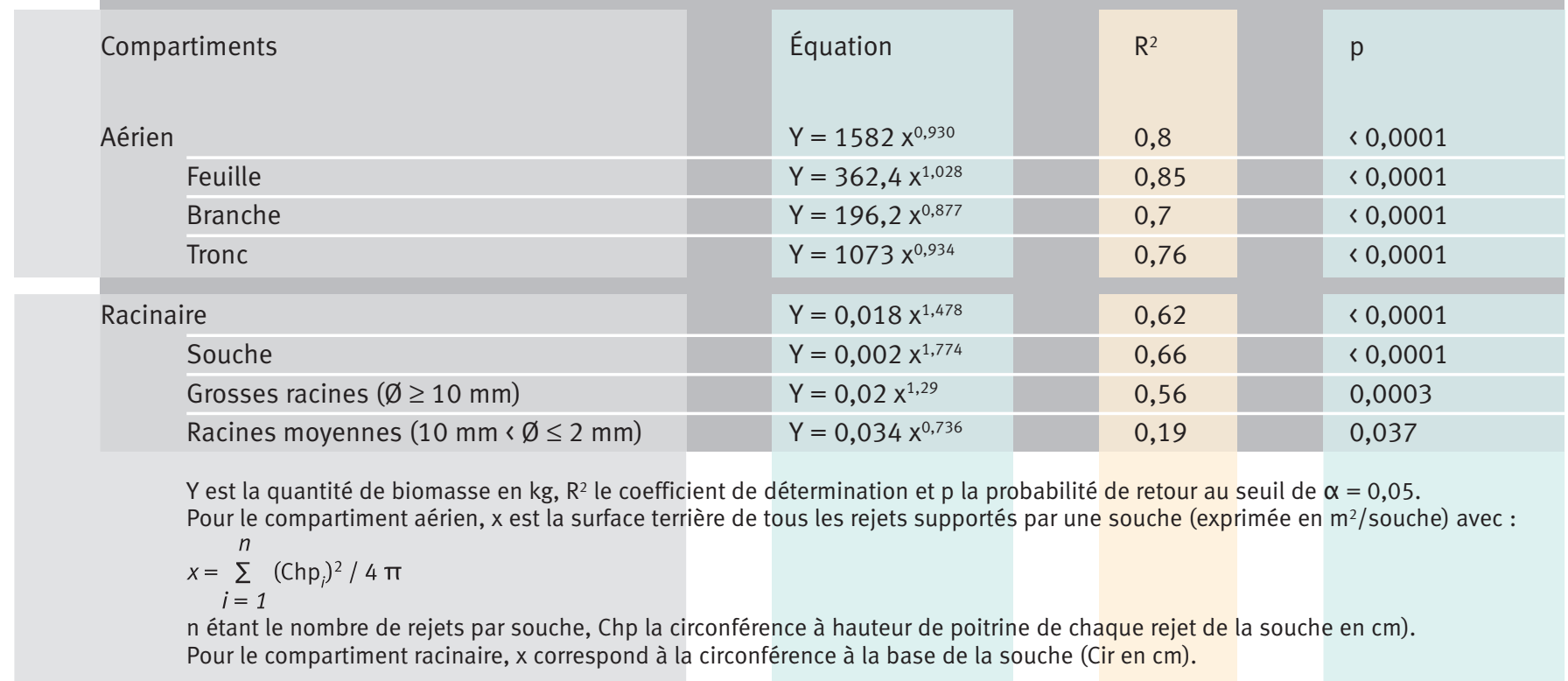




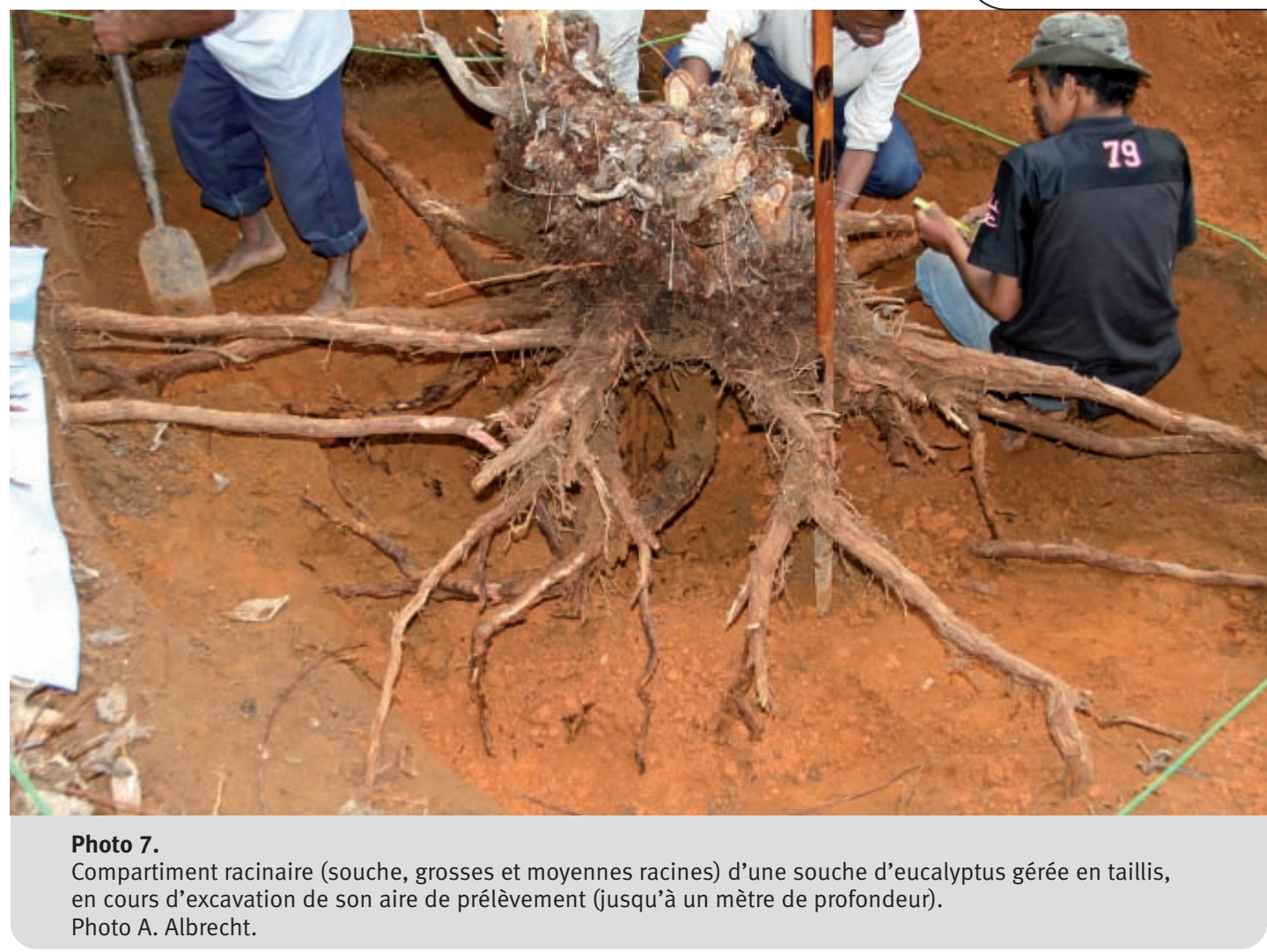

sières) et broyé à une épaisseur de $200 \mu \mathrm{m}$. La teneur en C organique a été mesurée par combustion par voie sèche (microanalyseur Carlo Erba 2000). Pour des sols présentant des éléments grossiers $(\varnothing>2 \mathrm{~mm})$, les les stocks de carbone $C_{\text {sol }}$ (en $\mathrm{Mg} \mathrm{C} / \mathrm{ha}$ ) sont calculés d'après la formule suivante (BATJES, 1996) :

Formule (1)

$C_{\text {sol }}=[C \times(100-\%$ en éléments grossiers $) \times$ Da x e $] / 10$

Avec :

- C : la teneur en carbone organique du sol d'éléments inférieurs à $2 \mathrm{~mm}$ (mg/g sol) ;

- \% en éléments grossiers : pourcentage de la fraction massique des éléments de plus de $2 \mathrm{~mm}$;

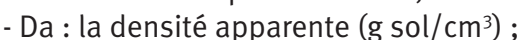

- e : épaisseur de l'horizon de sol (cm).

La formule (1) permet de passer d'une teneur à un stock.

Une correction a été apportée aux $C_{\text {sol }}$ calculés puisque les situations à comparer présentaient des profils de densité apparente différents (ELLERT, BETTANY, 1995 ; GIFFORD, RoDERICK, 2003). En conséquence, les $C_{\text {sol }}$ ont été ramenés à une profondeur de référence de $0-30 \mathrm{~cm}$ et à une masse équivalente de terre fixée à $4000 \mathrm{Mg}$ sol/ha (TURNER, LAMBERT, 1999 ; PAUL et al., 2002 ; LANDAIS, 2003).

\section{Étude de la composition isotopique du carbone de la matière organique du sol}

Selon les données disponibles, seules 14 parcelles sur les 57 sont concernées par l'étude isotopique sous différents modes d'usage (deux sous pseudo-steppe, quatre sous culture/jachère, huit sous eucalyptus âgées de 50 à 111 ans). La technique de traçage par l'abondance naturelle utilise la différence naturelle du rapport des deux isotopes stables du $C$ entre les végétations en $C_{4}$ (graminées et espèces herbacées) et en $C_{3}$ (dicotylédones ou espèces arborées), ceci par rapport à un standard international (BALESDENT, 1991). Cette technique offre ainsi un traceur naturel des changements d'écosystèmes et de la dynamique du $\mathrm{C}_{\text {sol }}$. À partir du $\delta^{13} \mathrm{C}$ de l'échantillon de sol, et connaissant les valeurs de $\delta^{13} \mathrm{C}$ de chaque végétal considéré, cette technique permet d'estimer la proportion du $C$ hérité de la végétation originelle $\left(C_{4}\right.$ savane/pseudo-steppe dans le cas présent) et du C dérivé de la nouvelle végétation sur place ( $\mathrm{C}_{3}$ eucalyptus) (BERNoux et al., 1998). Les valeurs de $\delta^{13} \mathrm{C}$ mentionnées dans la littérature ont été reprises : - $29 \%$ o pour la litière et racine de l'eucalyptus (TROUVÉ et al., 1994 ; MAQUĖRE et al., 2008) et calculée à - 17,6 \%o pour la pseudo-steppe. Cette valeur de $\delta^{13} \mathrm{C}$ de la pseudo-steppe a été obtenue en tenant compte à la fois de l'abondance des plantes qui la constituent et de leurs valeurs de $\delta^{13} \mathrm{C}$ qui sont de $-12 \%$ o pour les espèces graminéennes Andropogon brazzae, Loudetia spp. (TROUVÉ et al., 1994) et de - 28,7\%o pour Philippia sp. - valeur de $\delta^{13} \mathrm{C}$ de Arctostaphylon sp. selon SMITH et EPSTEIN (1971), une espèce de la même famille que Philippia sp..

Ainsi pour un site qui a initialement porté une végétation $\mathrm{C}_{4}$, la proportion de carbone d'origine $\mathrm{C}_{3}$ actuellement sur le site est calculée par : 


\section{Résultats et discussion}

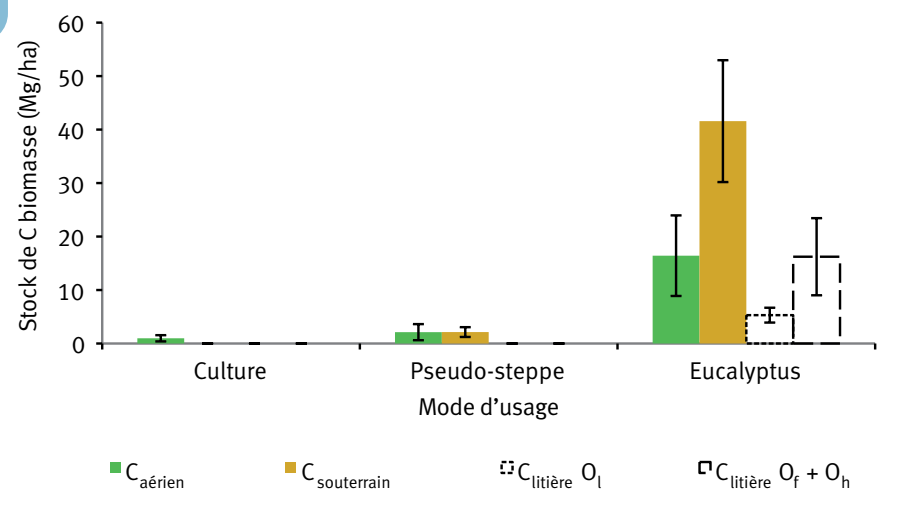

Figure 1.

Stock de carbone ( $\mathrm{Mg} \mathrm{C} / \mathrm{ha}$ ) dans le compartiment de la biomasse des divers modes d'usage des terres sur les Hautes Terres centrales de Madagascar. Pour les taillis d'eucalyptus : $C_{\text {aérien }}$ regroupe les feuilles, les branches et les troncs de tous les rejets d'une souche ; $C_{\text {racinaire }}$ regroupe les grosses $(\varnothing \geq 10 \mathrm{~mm})$ et moyennes $(2 \mathrm{~mm} \leq \varnothing<10 \mathrm{~mm})$ racines ; $\mathrm{C}_{\text {litière }} \mathrm{O}_{1}$ représente la litière de l'année et $\mathrm{C}_{\text {litière }} \mathrm{O}_{f}+\mathrm{O}_{h}$ celle déjà fragmentée et humifiée. Les barres d'erreurs présentent les écarts-types des stocks mesurés.

Formule (2)

$C_{\text {tot }}=C_{C_{4}}+C_{C 3}$

Formule (3)

$C_{\text {tot }} \delta_{\text {tot }}=C_{\mathrm{C}_{4}} \times \delta_{\mathrm{C}_{4}}+\mathrm{C}_{\mathrm{C} 3} \times \delta_{\mathrm{C} 3}$

Avec :

- $C_{\text {tot }}$ et $\delta_{\text {tot }}$ : la teneur en carbone total dans le sol portant la végétation $C_{3}$ et son $\delta_{13} C$;

- $C_{\mathrm{C}_{3}}$ et $\delta_{\mathrm{C}_{3}}$ : la teneur en carbone provenant du carbone incorporé par l'eucalyptus et le $\delta_{13} \mathrm{C}$ de la litière d'eucalyptus ;

- $C_{C_{4}}$ et $\delta_{C_{4}}$ : la teneur en carbone provenant du carbone hérité de l'ancienne végétation et le $\delta_{13} C$ de la plante qui l'a constitué.

À partir de la transformation de la formule (2) et de l'application du résultat à la formule (3), est obtenue la formule (4) :

Formule (4)

$\mathrm{C}_{\mathrm{C} 3} / \mathrm{C}_{\text {tot }}=\left(\delta_{\text {tot }}-\delta_{\mathrm{C}_{4}}\right) /\left(\delta_{\mathrm{C} 3}-\delta_{\mathrm{C} 4}\right)$

Par la suite, les résultats issus de ce dernier rapport ont été appliqués à la formule (1) :

$C_{\mathrm{sol}}=[\mathrm{C} \times(100-\%$ en éléments grossiers $) \times \mathrm{Da} \times \mathrm{e}] / 10$

pour calculer les stocks de carbone dérivés soit de l'eucalyptus ou de la pseudo-steppe.

\section{Analyses statistiques}

Une analyse de la variance (Anova) a été effectuée afin de tester l'effet des modes d'usage des terres sur les stocks de C. Pour la chronoséquence de plantations d'eucalyptus, nous avons aussi étudié les relations entre la durée de plantation et les stocks de C mesurés dans les différents compartiments, en calculant un coefficient de détermination $R^{2}$ et sa probabilité de significativité $p$ au seuil de $\alpha=0,05$.

\section{Les quantités de carbone de la biomasse (aérienne, racinaire, litière)}

La répartition de la quantité de $\mathrm{C}$ dans les différents compartiments de la biomasse change d'un mode de gestion à l'autre, avec une plus large quantité contenue dans la biomasse de l'eucalyptus (figure 1). En effet, les stocks de C de l'ensemble des parties aériennes et racinaires sont de 0,1 à $5,1 \mathrm{Mg} \mathrm{C} / \mathrm{ha}$, 2,1 à $7,2 \mathrm{Mg} \mathrm{C} /$ ha et 19 à $90 \mathrm{Mg} \mathrm{C} /$ ha respectivement pour la culture, la pseudo-steppe et les plantations d'eucalyptus. En considérant un à un les compartiments et en comparaison avec les autres modes d'usage, il apparaît que les eucalyptus renferment jusqu'à huit fois plus de $\mathrm{C}$ dans leur compartiment aérien (avec 16,4 $\pm 7,5 \mathrm{Mg} \mathrm{C} / \mathrm{ha}$ ) et environ vingt fois plus dans le compartiment racinaire (avec 41,6 $\pm 11,4 \mathrm{Mg} \mathrm{C/ha)} \mathrm{(tableau} \mathrm{III).} \mathrm{C'est}$ principalement la partie racinaire qui confère aux eucalyptus cette grande capacité de séquestration de C puisqu'elle représente environ 70 \% du C de la biomasse végétale grâce aux réservoirs constitués par la souche et les grosses racines. Néanmoins, il est à noter que les coefficients de détermination associés aux relations allométriques sont assez faibles par rapport à ceux trouvés dans la littérature pour des écosystèmes moins âgés et homogènes (SAINT-ANDRÉ et al., 2005), notamment pour les grosses et moyennes racines; ce qui témoigne de la grande variabilité racinaire affichée par l'écosystème étudié.

À ces stocks de $C$ dans la biomasse vivante des eucalyptus viennent s'ajouter ceux du compartiment de la litière au niveau duquel les couches $\mathrm{O}_{l}$ et $\mathrm{O}_{\mathrm{f}}+\mathrm{O}_{\mathrm{h}}$ contiennent respectivement environ 5,3 et $16,2 \mathrm{MgC} /$ ha (versus $0 \mathrm{Mg} \mathrm{C} /$ ha pour les autres modes d'usage). Ces valeurs paraissent être supérieures à celles trouvées dans d'autres études: $2 \mathrm{Mg}$ C/ha pour un peuplement d'E. camaldulensis au Cameroun (HARMAND et al., 2004) et 3,3 Mg C/ha sous des hybrides d'eucalyptus au Congo (LACLAU, 2001). Effectivement, le mode de gestion y est différent (taillis non âgés comme dans notre étude) et la couche $\mathrm{O}_{\mathrm{f}}+\mathrm{O}_{\mathrm{h}}$ n'est pas considérée.

Par ailleurs, les stocks de $\mathrm{C}$ de la savane $(\approx 2,1 \mathrm{Mg} \mathrm{C} / \mathrm{ha}$ chacun pour les parties aérienne et racinaire) sont proches des valeurs trouvées dans d'autres études $(1,7$ et $4,1 \mathrm{Mg} \mathrm{C} /$ ha respectivement pour l'aérien et le racinaire ; LACLAU, 2001). Pour les eucalyptus, les stocks mesurés sont similaires à ceux obtenus par ZEWDIE et al. (2009), qui sont de l'ordre de 16,1 Mg C/ha dans le compartiment aérien d'un taillis d'E. globulus dont les rejets sont âgés de 4 ans. Ce qui ne s'accorde pas avec les stocks mesurés sur des plantations d'eucalyptus gérées en futaie (LACLAU, 2001 ; HARMAND et al., 2004) au niveau desquelles la partie aérienne du tronc compte $21-32 \mathrm{Mg} \mathrm{C} /$ ha et la partie racinaire seulement de 4-7 Mg C/ha. Le fonctionnement biologique des systèmes en taillis qui présentent des lignotubers leur permettant de rejeter pourrat expliquer ces différences de répartition du stock de $\mathrm{C}$ dans les plantations d'eucalyptus. Les lignotubers sont en fait des organes producteurs racinaires qui permettent à la souche de produire de nouvelles pousses une fois la partie aérienne détruite (JACOBS, 1982). Ainsi, après chaque coupe, la souche émet des rejets qui la recouvrent et ajoutent à chaque repousse une certaine quantité de cals, et parallèlement des racines secondaires croîtraient entre chaque rotation (WILDY, PATE, 2002). 
Les quantités de carbone de la matière organique du sol

\section{Stocks de $\mathrm{C}_{\text {sol }}$ et modes d'usage des terres ${ }^{1}$}

Les résultats de mesures de stocks moyens de $\mathrm{C}_{\text {sol }}$ sur 0$30 \mathrm{~cm}$ à masse équivalente de sol montrent qu'en général les cultures présentent les stocks significativement les plus faibles (avec 74,2 $\pm 15,6 \mathrm{Mg} \mathrm{C} / \mathrm{ha}$ ). Les plantations d'eucalyptus (tous âges de plantation confondus) et la pseudo-steppe renferment des $C_{\text {sol }}$ plus élevés et ne sont pas significativement différents entre eux $(84,8 \pm 23 \mathrm{Mg} \mathrm{C} /$ ha et $91,7 \pm 18 \mathrm{Mg} \mathrm{C} /$ ha respectivement). Ces valeurs mettent en exergue l'importance du changement de mode d'usage des terres quant aux variations du $\mathrm{C}_{\text {sol }}$, comme ce qui est mentionné dans la littérature (TURNER, LAMBERT, 1999 ; POST et al., 2001 ; Houghton, GoOdale, 2004 ; JANDL et al., 2007), selon laquelle :

- la conversion des terres sous végétation herbacée en parcelle de culture conduit à une réduction du stock de $C_{\text {sol }}$ jusqu'à $50 \%$ ou plus en fonction de la pluviosité et de la durée depuis laquelle la pratique culturale a été effectuée (GUO, GIFFORD, 2002 ; ZINGORE et al., 2005) ; dans notre étude, cette réduction est en moyenne de $19 \%$ (figure 2) malgré une mise en culture de longue date, cela tenant probablement à l'apport de fumure à base de litière d'eucalyptus sur ces parcelles ; - la conversion des terres sous végétation herbacée en plantation forestière, notamment les feuillus (et Eucalyptus sp.), n'affecte pas le $C_{\text {sol }}(G \cup 0$, GIFFORD, 2002) car ils ont une plus grande capacité d'accumuler le $C_{\text {sol }}$ que les conifères ; cependant, des contradictions sont aussi mentionnées, selon

Tableau III.

Répartition en pourcentage du stock de carbone (C) dans les différents compartiments des plantations d'eucalyptus sur les Hautes Terres centrales de Madagascar.

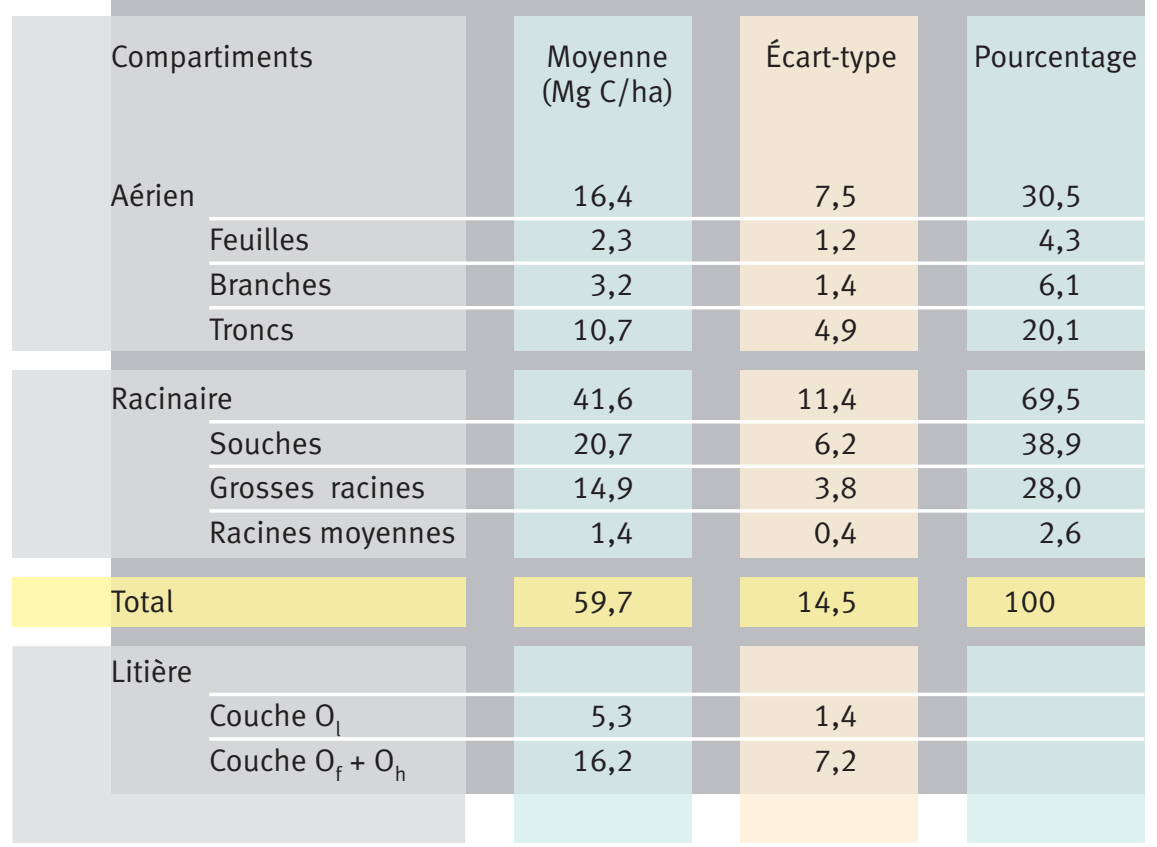

${ }^{1}$ Un aperçu de ces résultats est inclus dans la figure 4. lesquelles l'afforestation conduit à une diminution, une augmentation ou à un effet négligeable sur le $\mathrm{C}_{\text {sol }}$ (LAGANIÈRE et al., 2009). Malgré cela, le boisement montre une tendance générale à une perte du stock de $C_{\text {sol }}$ durant les premières années de plantation, suivie d'un retour graduel au niveau initial, puis d'une augmentation pour générer un gain net (PAul et al., 2002 ; Vesterdal et al., 2002).

\section{L'étude isotopique du $\mathrm{C}_{\text {sol }}$}

Même si des parcelles sous culture ont été considérées, l'étude isotopique s'est essentiellement portée sur les situations en système originel (pseudo-steppe) et en système planté en eucalyptus. En effet, les valeurs de $\delta^{13} \mathrm{C}$ des sols sous culture sont toujours les mêmes ( $-19,2 \%$ ) de 0 à $40 \mathrm{~cm}$ de profondeur (figure 2 a). Pour ces valeurs de $\delta^{13} \mathrm{C}$, des différences significatives existent seulement pour les vingt premiers centimètres du sol ; elles vont de - 24,2 à - 21,1\%。 sous eucalyptus (tous âges confondus) et de - 18,5 à - 17,6 \%o sous pseudo-steppe, respectivement dans les horizons 0-10 et $10-20 \mathrm{~cm}$. En considérant la chronoséquence sur les cinq parcelles d'eucalyptus (50 à 111 ans), aucune différence n'est constatée le long du profil 0-40 cm ; ces valeurs de $\delta^{13} \mathrm{C}$ sont proches de celles mesurées par TROuvé et al. (1994) au Congo, avec la valeur de - 25,1 \%o dans l'horizon 0-5 cm. Parallèlement, les pourcentages de $C$ apporté par les eucalyptus ne prédominent qu'en surface, avec 58,3\% et $30,5 \%$ respectivement sur $0-10 \mathrm{~cm}$ et 10 $20 \mathrm{~cm}$ (figure $2 \mathrm{~b}$ ). L'incorporation dans le sol de la matière organique issue de l'eucalyptus se produit donc principalement dans l'horizon 0-20 cm sous les taillis d'eucalyptus des Htc de Madagascar. Guo et GIFFORD (2002) et MAQUĖRE et al. (2008) suggèrent que c'est la courte durée de la rotation qui limiterait l'incorporation en profondeur du $\mathrm{C}_{\text {eucalyptus }}$ dans le sol.

\section{Le stock total de carbone}

En considérant tous les compartiments (aérien, racinaire et sol) pour chaque mode d'usage des terres, il est montré que les eucalyptus présentent des stocks de C significativement élevés (150 $\pm 25,3 \mathrm{Mg} \mathrm{C} /$ ha ; figure 3), provenant de la contribution des compartiments racinaires (racines et sol). En effet, pour les plantations d'eucalyptus, racines et sol ensemble constituent $85 \%$ du stock de C du système, auxquels s'ajoutent les stocks de $\mathrm{C}$ des compartiments aérien et de la litière $\left(\mathrm{O}_{\mathrm{l}}\right)(11 \%$ et $4 \%$ chacun, versus $1-2 \%$ et $0 \%$ pour les autres modes d'usage). Par contre, dans les autres modes d'usage, c'est uniquement le sol qui constitue le principal compartiment pour séquestrer du C (plus de $95 \%$ ). 


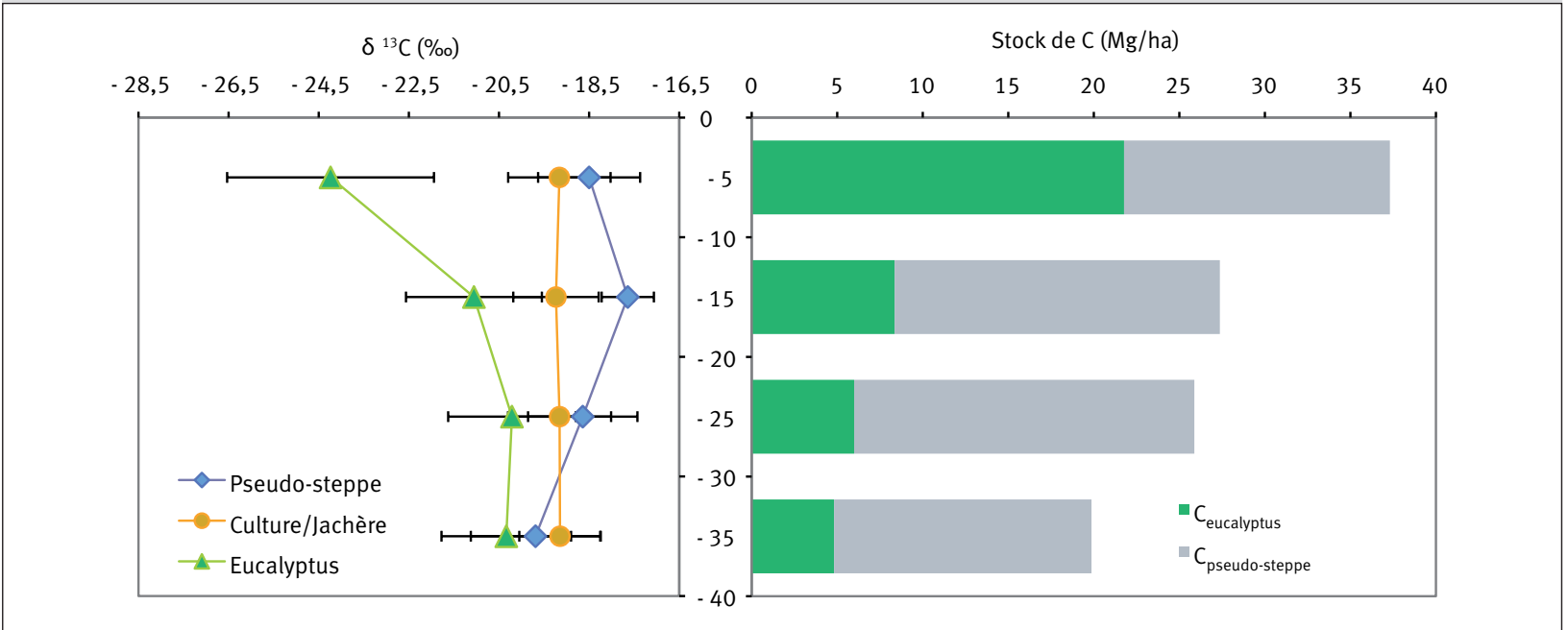

Figure 2.

(a) Profil de $\delta^{13} \mathrm{C}(\%)$ sous les différents modes d'usage des terres pour l'horizon $0-40 \mathrm{~cm}$.

(b) Contribution de l'eucalyptus dans la constitution du $\mathrm{C}_{\text {sol }}$.

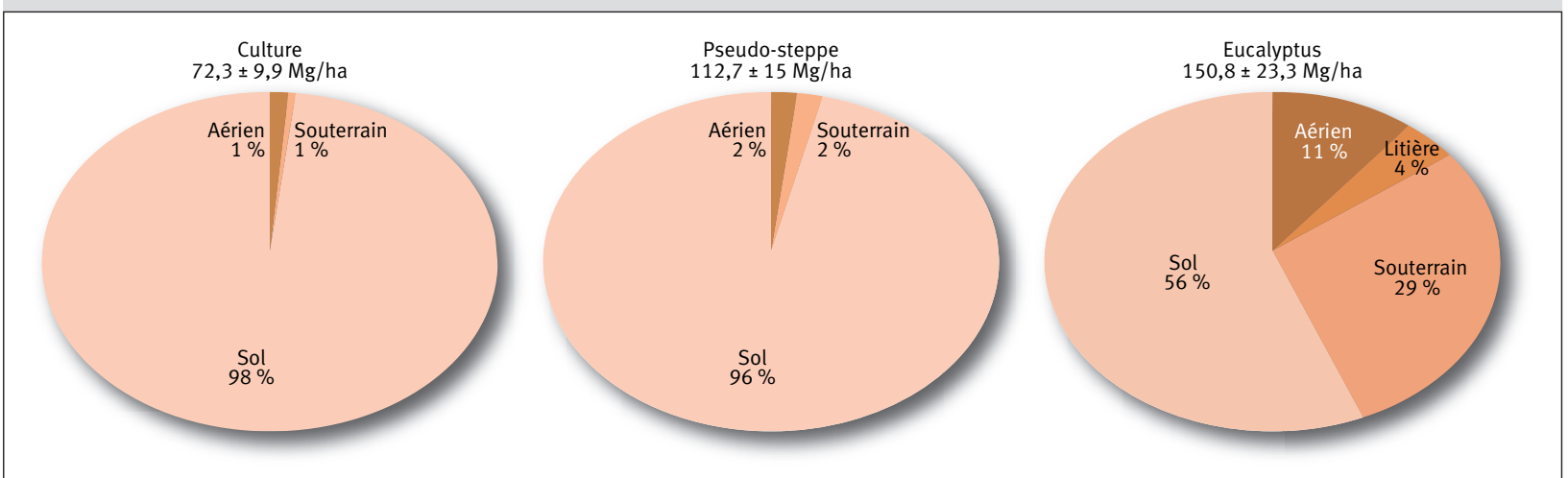

Figure 3.

Répartition du stock de carbone (en \%) dans les différents compartiments de chaque mode d'usage des terres des Hautes Terres centrales de Madagascar (les modes d'usage avec les mêmes lettres entre parenthèses ne présentent pas de différence significative au seuil de $\alpha=0,05$ ).

\section{L'évolution avec le temps des stocks de carbone des compartiments le séquestrant}

\section{Les relations entre stocks de carbone et temps}

En supposant que le compartiment aérien et la litière puissent être considérés comme «non permanents » (exportés suivant les pratiques locales), ce sont les racines et le sol qui constituent les compartiments « séquestrants ».

Les relations entre le stock de $C$ de chaque compartiment et la durée de plantation, présentées dans la figure 4, montrent qu'il existe une corrélation positive entre les stocks de $C$ racinaire et la durée de plantation ( $p=0,0003 ; n=44)$, avec environ $51 \%$ de la variabilité du stock racinaire expliquée par l'âge de plantation ; aucune corrélation significative n'est détectée entre les stocks de $C_{\text {sol }}$ et l'âge de plantation $\left(p=0,083 ; R^{2}=0,07\right.$; $\mathrm{n}=42$ ). La durée de plantation explique donc seulement une part de la variabilité des stocks de C à la fois dans le sol et les racines. D'autres paramètres pourraient contrôler le stock de $C$ des compartiments aérien et racinaire, tels que la densité de plantation, la texture du sol (FELler, BeARE, 1997 ; CROw, Hous-
TON, 2004), qui mériteraient d'être considérés, ceci non seulement dans le but de pouvoir les apprécier plus précisément avec le temps mais aussi en vue du développement des activités de rente carbone (GUTIÉRREZ et al., 2006).

\section{Les variations de stocks de carbone}

Compte tenu de la durée de plantation, les plantations d'eucalyptus sur les Htc malgaches présentent une diminution du stock de $\mathrm{C}_{\text {sol }}$ jusqu'à $31 \%$ du $\mathrm{C}$ initial pour les parcelles de moins de 50 ans et un gain jusqu'à 3,6 à 39,5\% pour les parcelles de plus de 100 ans (partie gauche de la figure 4). Selon des études récentes, le stock de $C_{\text {sol }}$ diminue durant les dix premières années de plantation (MARIN-SPIOTTA et al., 2009) jusqu'à 5,6 \% et augmente jusqu'à 6,1 à $18 \%$ les vingt années suivantes (LAGANIÈRE et al., 2009). D’après PAul et al. (2002), le stock de $C_{\text {sol }}$ de la végétation herbacée initiale est atteint 10-22 ans après l'afforestation et au cours des 40 années après la conversion, le $C_{\text {sol }}$ de la plantation étant supérieur au $\mathrm{C}_{\text {sol }}$ de l'antécédent cultural. 


\section{Conclusion}

Par la suite, les données sur les valeurs moyennes des stocks de $C_{\text {sol }}$ sous pseudo-steppe et culture $(91,7$ et $74,2 \mathrm{Mg} / \mathrm{ha}$ respectivement, partie droite de la figure 4) ont été reprises pour les analyser parallèlement avec la figure 4. Il apparaît alors que, après plus de 100 ans de plantation forestière, la variation du stock de $C_{\text {sol }}$ entre culture et eucalyptus est importante $\left(\Delta \mathrm{C}_{\text {sol }}\right.$ culture $\left.\approx 25 \mathrm{Mg} / \mathrm{ha}\right)$, tandis que la variation entre pseudosteppe et eucalyptus reste faible $\left(\Delta C_{\text {sol }}\right.$ pseudo-steppe $\approx$ $5 \mathrm{Mg} / \mathrm{ha}$ ). De plus, en raison des conditions de pratiques locales (coupe rase et exploitation de la litière à chaque fin de rotation de 5 ans), les taillis d'eucalyptus des Htc malgaches semblent demander plus de temps $(\approx 80$ ans et plus) pour le recouvrement du $\mathrm{C}_{\text {sol }}$ initial sous pseudo-steppe. En revanche, un gain net est observé dans le compartiment racinaire en comparaison avec la végétation originelle correspondant à un $\Delta C_{\text {racinaire }}$ pseudo-steppe de l'ordre de $45 \mathrm{Mg} / \mathrm{ha}$. Ce constat sur le $\mathrm{C}_{\text {sol }}$ a aussi été mentionné dans une étude sur le rôle du stock de $C_{\text {sol }}$ d'un écosystème similaire en Australie (plantation d'Eucalyptus globulus source de biomasse pour la bioénergie renouvelable) par Cowie et al. (2006). En effet, ils ont conclu que, ces systèmes de plantation à courte rotation pourraient amener à une faible diminution du stock de $\mathrm{C}_{\text {sol }}$. Cependant, ils ont aussi mentionné que cette baisse du stock de $C_{\text {sol }}$ peut être négligeable en comparaison avec la contribution des systèmes bioénergies vers l'atténuation des Ges, cela, à travers l'évitement des émissions de combustibles fossiles.
Les fonctions usuelles socio-économique et environnementale des taillis d'eucalyptus des Hautes Terres centrales malgaches en termes de fourniture de bois de chauffe, d'énergie et de diminution des pressions sur les forêts naturelles sont indéniables. De plus, la présente étude a montré leur importance écologique en termes de capacité de séquestration de C. La méthodologie développée a permis d'estimer de façon précise le stock de $\mathrm{C}$ de tous les compartiments, particulièrement le compartiment des racines. Ce dernier n'est habituellement évalué qu'en utilisant un ratio du stock de C du compartiment aérien, moins précis mais plus facilement quantifiable. Les résultats ont montré que les plantations d'eucalyptus stockent une quantité

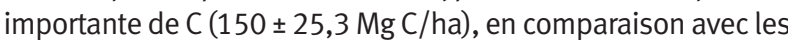
autres modes d'usage des terres considérés (rotation culture/jachère, savane/pseudo-steppe). Cette fonction puits de carbone est notamment due aux contributions considérables de la biomasse et de la matière organique des compartiments racinaire et du sol (29 et $56 \%$ du stock total de C respectivement). L'étude de la relation des stocks de $\mathrm{C}$ dans ces deux compartiments avec la durée de plantation a permis de comprendre leur évolution avec le temps, mais également de constater que ce facteur n'explique qu'une part de la variabilité des stocks. Il est donc nécessaire de considérer d'autres facteurs qui vont contrôler ces capacités de séquestration de C. Néanmoins, cette étude fournit déjà des éléments importants pour estimer la variation du stock de C lors d'un reboisement.

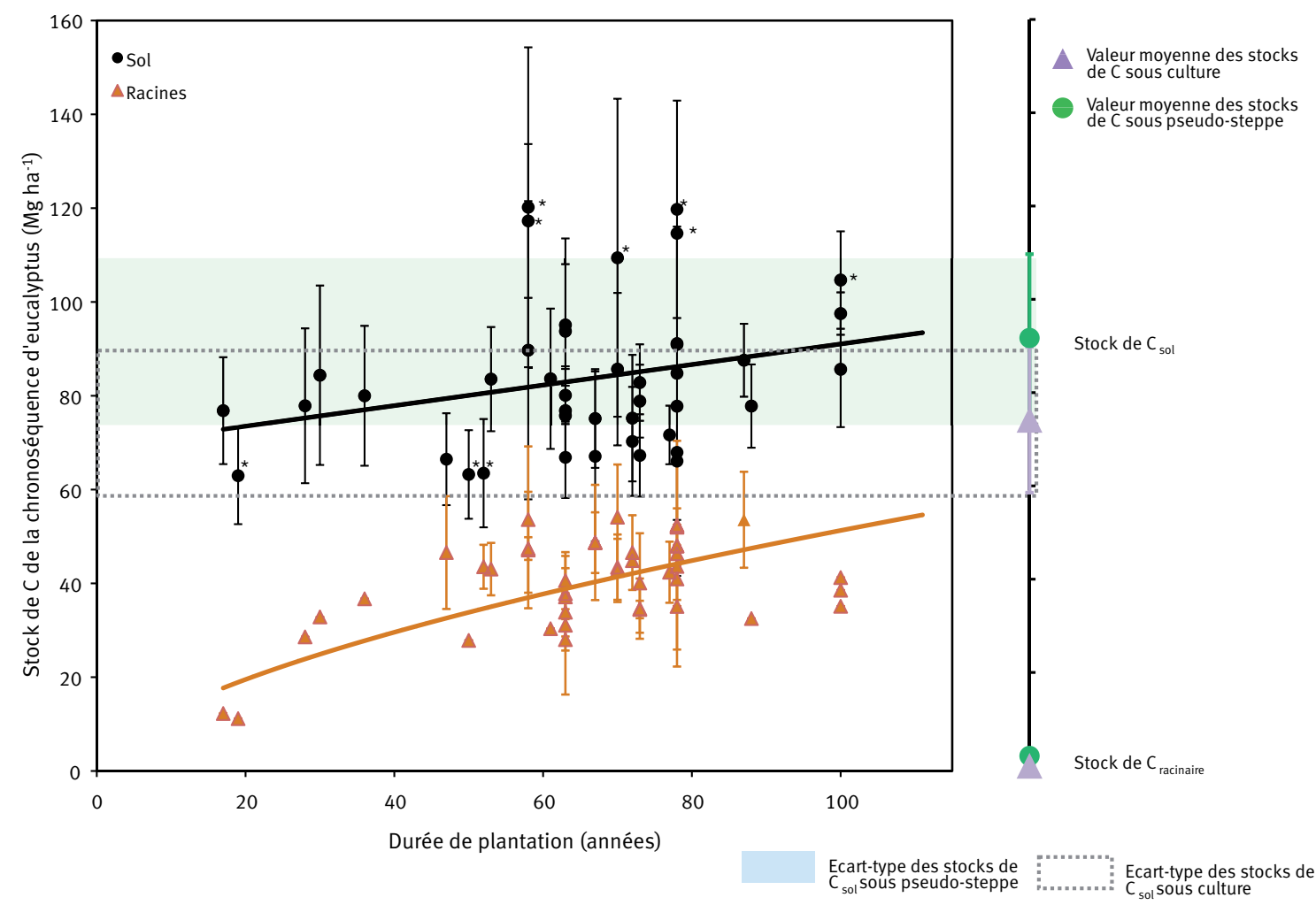

Figure 4.

Évolution des stocks de carbone ( $\mathrm{Mg} \mathrm{C} /$ ha) des compartiments séquestrants (racinaire et sol) des taillis d'eucalyptus des Hautes Terres centrales de Madagascar avec leur durée de plantation. Les barres d'erreur représentent les écartstypes des stocks au sein de chaque parcelle. Les individus accompagnés d'un astérisque présentent des différences significatives avec les parcelles sous pseudo-steppe au seuil de $\alpha=0,05$.

Expressions des équations de régression $y=0,22 x+69,1$ pour le $C_{\text {sol }}\left(R^{2}=0,073 ; p=0,083 ; n=42\right)$, en noir, et $y=3,23 x^{0,6}$ exposant pour le $C_{\text {racinaire }}\left(R^{2}=0,51 ; p=0,0003 ; n=44\right)$, en orange. 
Ces résultats trouvent leur intérêt avec les enjeux et besoins en matière de connaissances internationales ou nationales sur l'appréciation rapide et fiable des capacités de fixation de $\mathrm{C}$ atmosphérique des plantations forestières, en particulier des taillis âgés dans le cadre de cette étude. Ces évaluations précises permettront à Madagascar de suivre les avancées des négociations internationales sur la Convention-cadre des Nations unies sur le changement climatique (Ccnucc) et d'en bénéficier. Effectivement, les résolutions post-Kyoto offriront des options prometteuses avec le processus de Réduction des émissions liées à la déforestation et à la dégradation des forêts (Redd) des gaz à effet de serre, mais aussi lors du développement de projets du Mécanisme de développement propre sur d'autres zones d'afforestation.

\section{Références}

BALESDENT J., 1991. Estimation du renouvellement du carbone des sols par mesure isotopique ${ }^{13} \mathrm{C}$ : précision, risque de biais. Cahiers de l'Orstom, série Pédologie, 26 : 315-326.

BATJES N. H., 1996. Total carbon and nitrogen in the soils of the world. European Journal of Soil Science, 47: 151-163.

BERNOUX M., CERRI C. C., NEILL C., DE MORAES J. F. L., 1998. The use of stable carbon isotopes for estimating soil organic matter turnover rates. Geoderma, 82: 43-58.

BERTRAND A., 1999. La dynamique séculaire des plantations paysannes d'Eucalyptus sur les Hautes Terres malgaches. Le Flamboyant, 49 : 45-48.

BOURGEAT F., ZEBROWSKI C., 1973. Relations entre le relief, les types de sols et leurs aptitudes culturales sur les Hautes Terres Malgaches. Cahiers de l'Orstom, série Biologie, 19 : 23-41.

CARRIÈRE S. M., RANDRIAMBANONA H., 2007. Biodiversité introduite et autochtone : antagonisme ou complémentarité ? Le cas de l'eucalyptus à Madagascar. Bois et forêts des tropiques, $292:$ 5-21.

COWIE A. L., SMITH P., JOHNSON D., 2006. Does soil carbon loss in biomass production systems negate the grenhouse benefits of bioenergy ? Mitigation and Adaptation Strategies for Global Change, 11: 979-1002.

CROW P., HOUSTON T. J., 2004. The influence of soil and coppice cycle on the rooting habit of short rotation poplar and willow coppice. Biomass and Bioenergy, 26: 497-505.

DALAL R. C., ALLEN D. E., LIVESLEY S. J., RICHARDS G., 2008. Magnitude and biophysical regulators of methane emission and consumption in the Australian agricultural, forest, and submerged landscapes : a review. Plant Soil, 309: 43-76.

ELLERT B. H., BETTANY J. R., 1995. Calculation of organic matter and nutrients stored in soils under contrasting management regimes. Canadian Journal of Soil Science, 75: 529-538.

FAO, 2001. Soil carbon sequestration for improved land management. Rome, Italie, Fao, World Soil Resources Reports 96, 74 p.
FAO, 2006. World reference base for soil resources 2006. A framework for international classification, correlation and communication. Rome, Italie, Fao, World Soil Resources Reports 103, 145 p.

FELLER C., BEARE M. H., 1997. Physical control of soil organic matter dynamics in the tropics. Geoderma, 79: 69-116.

FEST B. J., LIVESLEYS. J., DRÖSÏER M., VAN GORSELE E., ARNDT S. K., 2009. Soil-atmosphere greenhouse gas exchange in a cool, temperate Eucalyptus delegatensis forest in south-eastern Australia. Agricultural and Forest Meteorology, 149: 393-406.

GIFFORD M. R., RODERICK M. L., 2003. Soil carbon stocks and bulk density: spatial or cumulative mass coordinates as a basis of expression. Global Change Biology, 9: 1507-1514. GUÉNEAU P., 1969. L'Eucalyptus robusta à Madagascar. Bois et forêts des tropiques, 124 : 53-65.

GUO L. B., GIFFORD M., 2002. Soil carbon stocks and land use: a meta analysis. Global Change Biology, 8: 345-360.

GUTIÉRREZ V. H., ZAPATA M., SIERRA C., LAGUADO W., SANTACRUZ A., 2006. Maximizing the profitability of forestry projects under the Clean Development Mechanism using a forest management optimization model. Forest Ecology and Management, 226 (1-3): 341-350.

HARMAND J.-M., NJITI C. F., BERNHARD-REVERSAT F., PUIG H., 2004. Aboveground and belowground biomass, productivity and nutrient accumulation in tree improved fallows in the dry tropics of Cameroon. Forest Ecology and Management, 188 (1-3): 249-265.

HOUGHTON R. A., GOODALE C. L., 2004. Effects of Land-Use Change on the Carbon Balance of Terrestrial Ecosystems. In: AGU Chapman Conference, Santa Fe, États-Unis, 14 juin 2003. Washington, États-Unis, The American Geophysical Union, Geophysical Monograph Series 153, p. 85-98.

IPCC, 2006. Agriculture, Forestry and Other Land Use. In : Eggleston S., Buendia L., Miwa K., Ngara T., Tanabe K. (éd.). 2006 IPCC Guidelines for National Greenhouse Gas Inventories, vol. 4. Hayama, Kanagawa, Japon, Institute for Global Environment Strategies.

IPCC, 2007. Climate Change 2007 : The Physical Science Basis. Contribution of Working Group I to the Fourth Assessment Report of the Intergovernmental Panel on Climate Change. Cambridge, Royaume-Uni, Cambridge University Press, 996 p. JACOBS M. R., 1982. Les eucalyptus dans le reboisement. Rome, Italie, Fao, coll. Fao Forests, 753 p.

JANDL R., VESTERDAL L., OLSSON M., BENS O., BADECK F., ROCK R., 2007. Carbon sequestration and forest management. CAB Reviews: Perspectives in Agriculture, Veterinary Science, Nutrition and Natural Resources, 2, $n^{\circ} 17,16 \mathrm{p}$.

LACLAU J.-P., 2001. Dynamique du fonctionnement minéral d'une plantation d'eucalyptus. Effets du reboisement sur un sol de savane du littoral congolais; conséquences pour la gestion des plantations industrielles. Doctorat en sciences de la vie, Institut national agronomique Paris-Grignon, France, 175 p. 
LAGANIÈRE J., ANGERS, D., PARÉ D., 2009. Carbon accumulation in agricultural soils after afforestation : a meta-analysis. Global Change Biology, 16 (1): 439-453.

LAL R., 2005. Forest soils and carbon sequestration. Forest Ecology and Management, 220 (1-3): 242-258.

LANDAIS D., 2003. Études quantitatives et qualitatives du carbone séquestré dans le sol après afforestation au Congo Brazzaville. Mémoire de stage Dess en sciences de l'environnement, université Louis Pasteur, Strasbourg, France, 33 p.

LIVESLEY S. J., KIESE R., MIEHLE P., WESTON C. J., BUTTERBACH-BAHL K., ARNDT S. K., 2009. Soil-atmosphere exchange of greenhouse gases in a Eucalyptus marginata woodland, a clover-grass pasture, and Pinus radiata and Eucalyptus globulus plantations. Global Change Biology, 15 (2): 425-440.

LOCATELLI B., LESCUYER G., 1999. Rôle et valeur des forêts tropicales dans le changement climatique. Bois et forêts des tropiques, $260: 5-18$.

MAQUĖRE V., LACLAU J. P., BERNOUX M., SAINT-ANDRÉ L., GONCALVES L. M., CERRI C. C., PICCOLO M. C., RANGER J., 2008. Influence of land use (savanna, pasture, Eucalyptus plantations) on soil carbon and nitrogen stocks in Brazil. European Journal of Soil Science, 59 (5): 863-877.

MARIN-SPIOTTA E., SILVER W. L., SWANSTON C. W., OSTERTAG R., 2009. Soil organic matter dynamics during 80 years of reforestation of tropical pastures. Global Change Biology, 15 (6): 1584-1597.

MEYERS D., RAMAMONJISOA B., SĖVE J., RAJAFINDRAMANGA M., BURREN C., 2006. Étude sur la consommation et la production en produits forestiers ligneux à Madagascar. Antananarivo, Madagascar, Usaid, Groupe Irg, 101 p.

PAUL K. I., POLGLASE P. J., NYAKUENGAMA J. G., KHANNA P. K., 2002. Change in soil carbon following afforestation. Forest Ecology and Management, 168 (1-3): 241-257.

PELTIER R., FORKONG N. C., NTOUPKA M., MANLAY R., HENRY M., MORILLON V., 2007. Évaluation du stock de carbone et de la productivité en bois d'un parc à karités du Nord-Cameroun. Bois et forêts des tropiques, 294 : 39-50.

POST W. M., IZAURRALDE R. C., MANN L. K., BLISS N., 2001. Monitoring and verifying changes of organic carbon in soil. Climatic Change, 51: 73-99.

POST W. M., KNOWN K. C., 2000. Soil carbon sequestration and land-use change : processes and potential. Global Change Biology, 6: 317-327.

RANDRIANJAFY H., 1999. Les plantations d'Eucalyptus à Madagascar : superficie, rôle et importance des massifs. Projet Fao Gcp/Int/679/EC, Madagascar, 80 p.

ROBERT M., SAUGIER B., 2003. Contribution des écosystèmes continentaux à la séquestration du carbone. C. R. Géoscience, 335 : 577-595.
SAINT-ANDRÉ L., M'BOU A. T., MABIALA A., MOUVONDY W., JOURDAN C., ROUPSARD O., DELEPORTE P., HAMEL O., NOUVELLON Y., 2005. Age-related equations for above- and below-ground biomass of a Eucalyptus hybrid in Congo. Forest Ecology and Management, 205 (1-3): 199-214.

SANTANTONIO D., HERMAN R. K., OVERTOS W. S., 1977. Root biomass studies in forest ecosystems. Pedobiologia, 17: 1-31.

SMITH B. N., EPSTEIN S., 1971. Two categories of ${ }^{13} \mathrm{C} /{ }^{12} \mathrm{C}$ ratios of higher plants. Plant Physiology, 47 (3): 380-384.

TROUVÉ C., MARIOTTI A., SCHWARTZ D., GUILLET B., 1994. Soil organic carbon dynamics under eucalyptus and pinus planted on savannas in the Congo. Soil Biology and Biochemistry, 26: 287-295.

TURNER J., LAMBERT M., 1999. Change in organic carbon in forest plantation soils in Eastern Australia. Forest Ecology and Management, 133 (3): 231-247.

VESTERDAL L., RITTER E., GUNDERSEN P., 2002. Change in soil organic carbon following afforestation of former arable land. Forest Ecology and Management, 169 (1-2): 137-147.

WILDY D. T., PATE J. S., 2002. Quantifying above-and belowground growth response of the western Australian Oil Mallee, Eucalyptus kochii subsp. plenissima, to constracting decapitation regimes. Annals of Botany, 90: 185-197.

ZEWDIE M., OLSSON M., VERWIJST T., 2009. Above-ground biomass production and allometric relations of Eucalyptus globulus Labill. coppice plantations along a chronosequence in the central highlands of Ethiopia. Biomass and Bioenergy, 33: 421-428.

ZINGORE S., MANYAME C., NYAMUGAFATA P., GILLER K. E., 2005. Long-term changes in organic matter of woodland soils cleared for arable cropping in Zimbabwe. European Journal of Soil Science, 56: 727-736. 